\title{
SKOLEM FUNCTIONS IN NON-CLASSICAL LOGICS
}

\author{
TORE FJETLAND ØGAARD
}

\begin{abstract}
This paper shows how to conservatively extend theories formulated in non-classical logics such as the Logic of Paradox, the Strong Kleene Logic and relevant logics with Skolem functions. Translations to and from the language extended by Skolem functions into the original one are presented and shown to preserve derivability. It is also shown that one may not always substitute $s \doteq f_{A}(\bar{t})$ and $A(\bar{t}, s)$ even though $A(\bar{x}, y)$ determines the extension of a function.
\end{abstract}

Keywords:

Conservative extension, identity, Leibniz's law, non-classical logics, restricted universal quantification, Skolem functions, unique existential quantifiers.

\section{Contents}

1. Introduction 182

2. Definitions 184

3. Henkin constants 189

4. Skolem functions 192

5. Definable Skolem functions 195

6. Definable Skolem functions in Strong Kleene Logic 200

7. Definable Skolem functions in The Logic of Paradox 204

8. Definable Skolem functions in relevant logics 209

9. A brief glance at other logics 219

10. Summary 221

$\begin{array}{ll}\text { References } & 222\end{array}$

Appendix - Henkin axioms and Independence of Premise 223

Date: March 29, 2017.

Thanks to Zach Weber for encouraging me to write this paper and to the anonymous referee for providing helpful feedback.

Australasian Journal of Logic (14:1) 2017, Article no. 7 


\section{INTRODUCTION}

The axiom of choice entails that if every $A$ is related to at least one $B$, then there is a choice function which for every $A$ picks one such $B$. It does not, however, guarantee that one can introduce a function symbol for such a function. This is where Skolem functions come in handy. The now standard way of adding Skolem functions is to add Skolem axioms-formulas of the form $\forall \bar{x}\left(\exists y A(\bar{x}, y) \rightarrow A\left(\bar{x}, f_{A}(\bar{x})\right)\right)$. Such an axiom not only ensures that a choice function exists, but also introduces a name for it. ${ }^{1}$

In classical logic one can prove that adding Skolem axioms is conservative; if some formula in the language without Skolem functions is not derivable from a theory, then it remains non-derivable from the theory extended by Skolem axioms. This paper shows how to add Skolem functions to a theory when the theory is formulated in certain non-classical logics. It will turn out that it is rather trivial to do this provided the logic in question satisfies some basic principles. What is not trivial though is to show that Skolem functions can be useful.

In order for Skolem functions to be truly useful, one needs to know how one can validly reason with them. Skolem functions come in two different clothings-Skolem functions introduced for functional formulas and Skolem functions introduced for relational formulas where a formula $A(\bar{x}, y)$ is functional relative to some theory $\Theta$ if $\forall \bar{x} \exists ! y A(\bar{x}, y)$ is derivable from it, and relational if not. A Skolem function introduced for a functional formula is called a definable Skolem function. Reasoning with Skolem functions introduced for relational formulas has little to it; if $f_{A}(\bar{x})$ is introduced for a relational formula $A(\bar{x}, y)$, one should only expect the rule $s \doteq f_{A}(\bar{t}) \vdash A(\bar{t}, s)$ to be valid. The exception here is if the logic validates the rule $A(x / t), \neg A(x / s) \vdash t \not s$, E4: in that case one should furthermore expect $\neg A(\bar{t}, s) \vdash s \not f_{A}(\bar{t})$ to hold. This will easily be seen to be the case. Beyond this, however, there seems little more to it. For definable Skolem functions, however, there are at least two things one should expect: (1) that there is a way to translate back and forth between the original language and the language extended by definable Skolem functions which preserves derivability, and (2) that $A(\bar{t}, s)$ and $s \doteq f_{A}(\bar{t})$ are intersubstitutable. (2), however, requires that the laws of identity are in sync, so to speak, with the rest of the theory, and this I will show is not always the case.

\footnotetext{
${ }^{1}$ The Skolem axiom $\exists x A(x) \rightarrow A\left(c_{A}\right)$ is usually called a Henkin axiom. Many presentations of the completeness theorem for classical logic starts by expanding the theory in question with Henkin axioms. It is worth noting that neither Leon Henkin nor Thoralf Skolem made use of such axioms in, respectively, [10] and [21], but instead closed their theories under the rules $\exists x A(x) \vdash A\left(c_{A}\right)$ and $\forall \bar{x} \exists y A(\bar{x}, y) \vdash \forall \bar{x} A\left(\bar{x}, f_{A}(\bar{x})\right)$.
}

Australasian Journal of Logic (14:1) 2017, Article no. 7 
The main focus of this paper is on the so-called Strong Kleene Logic, $\mathbf{K}_{3}^{d}$, or more precisely a first-order version of that logic with identity, $\bar{\nabla} \mathbf{K}_{3}^{d}$, and a first-order version with identity of the Logic of Paradox, $\bar{\nabla} \mathbf{L} \mathbf{P}^{d}$. I will later also show some results which pertain to relevant logics and to the three-valued logics $\bar{\nabla} \mathbf{E}_{3}^{d}$ and $\bar{\nabla} \mathbf{R} \mathbf{M}_{3}^{d}$. The plan for the paper is as follows: section 2 introduces $\bar{\nabla} \mathbf{K}_{3}^{d}$ and $\bar{\nabla} \mathbf{L} \mathbf{P}^{d}$ and gives some basic definitions. Section 3 then shows how to add Henkin witnesses conservatively. Section 4 generalized this result so as to also cover Skolem functions. The main goal here is to show that $\bar{\nabla} \mathbf{K}_{3}^{d}$ can be conservatively extended by the rule $\forall \bar{x} \exists y A(\bar{x}, y) \vdash \forall \bar{x} A\left(\bar{x}, f_{A}(\bar{x})\right)$ which governs the introduction of Skolem functions. It will be plain to see that the proof works just as well for a wide class of logics, such as the four-valued logic $\bar{\nabla} \mathbf{F D E}{ }^{d}$, the $n \geq 2$-valued logics $\bar{\nabla} \mathbf{R} \mathbf{M}_{n}^{d}$ and Łukasiewicz logics, and intensional logics such as intuitionistic logic, relevant logics and the infinite-valued Łukasiewicz logic. That such a rule is possible to add conservatively corrects a proof, and solves a problem left open by Zach Weber in [24].

Section 5 then focuses on definable Skolem functions. The two standard ways of defining the unique existential quantifier $\exists$ ! $-\exists x(A(x) \wedge \forall y(A(y) \supset$ $y \doteq x)$ ) and $\exists x \forall y(A(y) \equiv y \doteq x)$ ) — come apart in $\bar{\nabla} \mathbf{K}_{3}^{d}$ due to the absence of the rule $A(x / t), \neg A(x / s) \vdash t \not s, \mathrm{E} 4$. The latter entails the former, but not vice versa. It is argued that unless one is ready to add E4 to the logic, then one ought to use the weaker version. However, I will show that if one does go for the weaker version, then although one can always conservatively substitute $s \doteq f_{A}(\bar{t})$ for $A(\bar{t}, s)$, this is not always the case when substituting $A(\bar{t}, s)$ for $s \doteq f_{A}(\bar{t})$. I also show that one can intersubstitute these formulas provided one uses the stronger definition of $\exists$ !. It is furthermore shown that there is a translation to and from the definable Skolem function extended language which preserves derivability in $\bar{\nabla} \mathbf{K}_{3}^{d}$.

Section 6 focuses on $\bar{\nabla} \mathbf{L} \mathbf{P}^{d}$. I show that the above mentioned translation also works for $\bar{\nabla} \mathbf{L} \mathbf{P}^{d}$, although not to the same extent: one can only preserve derivability when going from $\bar{\nabla} \mathbf{L} \mathbf{P}^{d}$ augmented by a Skolem rule for functional formulas to $\bar{\nabla} \mathbf{L} \mathbf{P}^{d}$, and not the other way around. It is also shown that one cannot in general substitute $A(\bar{t}, s)$ for $t \doteq f_{A}(\bar{t})$, nor $s \doteq f_{A}(\bar{t})$ for $A(\bar{t}, s)$, while retaining conservativeness. $\bar{\nabla} \mathbf{L} \mathbf{P}^{d}$ is for this reason deemed unfit for Skolem functions relative to both ways of defining $\exists$ !.

Section 7 focuses on relevant logics. These logics are also shown to be unfit for Skolem functions relative to several ways of defining $\exists$ !. The reason for this is basically due to the fact that the rule $A(x / t) \vdash \forall x(t \doteq x \rightarrow A)$, $\mathrm{E} 8$, is not derivable in these logics. I then give a brief comment on the possibility of utilizing the notion of relevant predication to solve the issue, but conclude that this theory is built upon the unwarranted assumption that 
E8 is relevantly permissible. The unfitness of these logics is mitigated by the fact that there is a translation which preserves derivability provided the logic in question validates Ackermann's $\delta$ rule $A \rightarrow(B \rightarrow C), B \vdash A \rightarrow$ $C$, has the Ackermann constant $\mathbf{t}$ and validates the strong, but relevantly permissible version of Leibniz's law $\forall x \forall y(A \rightarrow((x \doteq y \wedge \mathbf{t}) \rightarrow A(x / y)))$, E7.

Section 8 then briefly looks at the three-valued logics $\bar{\nabla} \mathbf{L}_{3}^{d}$ and $\bar{\nabla} \mathbf{R} \mathbf{M}_{3}^{d}$. The issue of how to define $\exists$ ! is then related to restricted universal quantification, and it is argued that at least one natural way of expressing Leibniz's law involves restricted universal quantification. The result concerning the translation for relevant logics is then generalized and it is shown that related logics which validates E7 and defines $\exists$ ! in terms of the restricted universal quantifier, also suffice for a derivability-preserving translation.

Relevant logics are like intuitionistic logic in that the the strong linearity rule $A \rightarrow \exists x B(x) \vdash \exists x(A \rightarrow B(x))$ called independence of premise fails in them. I show in the appendix that for a wide range of logics one can conservatively extend a theory by Henkin axioms if and only the theory in question is closed under this rule.

\section{Definitions}

This section presents the three-valued logics $\bar{\nabla} \mathbf{K}_{3}^{d}$ and $\bar{\nabla} \mathbf{L} \mathbf{P}^{d}$, and gives some basic definitions which will be used throughout this paper. As a starter, $\supset$ and $\equiv$ will be defined connectives:

\section{Definition 1.}

$$
\begin{aligned}
& A \supset B:=\neg A \vee B \\
& A \equiv B:=(A \supset B) \wedge(B \supset A)
\end{aligned}
$$


$\bar{\nabla} \mathbf{K}_{3}^{d}$ has the following rules: ${ }^{2}$

\begin{tabular}{lll} 
R1 & $A, B \vdash A \wedge B$ \\
R2 & $A \wedge B \vdash A$ and $A \wedge B \vdash B$ & \\
R3 & $A \vdash A \vee B$ and $B \vdash A \vee B$ & \\
R4 & $\neg \neg A \dashv A$ & \\
R5 & $\neg(A \wedge B) \dashv \neg A \vee \neg B$ & \\
R6 & $\neg(A \vee B) \dashv \neg A \wedge \neg B$ & \\
R7 & $A, \neg A \vee B \vdash B$ & (disjunctive syllogism) \\
\hline Q1 & $\forall x A \vdash A(x / t)$ & $t$ free for $x$ \\
Q2 & $\forall x(A \vee B) \vdash A \vee \forall x B$ & $x \notin F V\{A\}$ \\
Q3 & $A(x / t) \vdash \exists x A$ & $t$ free for $x$ \\
Q4 & $\neg \forall x A \dashv \exists x \neg A$ & \\
Q5 & $\neg \exists x A \dashv \forall x \neg A$ & \\
\hline E1 & $\forall x(x \doteq x)$ & $s \& t$ free for $x$ \\
E2 & $t \doteq s, A(x / t) \vdash A(x / s)$ &
\end{tabular}

I will also consider the logic $\bar{\nabla} \mathbf{K}_{3}^{d}[E 3]$ got by adding the following rule:

$$
\text { E3 } A(x / t) \vdash \forall x(t \doteq x \supset A) t \text { free for } x .
$$

Notably lacking from the above set of rules are universal generalization, reasoning by cases, and existential instantiation. These are sometimes included in the list of primitive rules of a logic as follows:

$$
\begin{array}{cll}
\text { RQ } \frac{\Gamma \vdash A(x / y)}{\Gamma \vdash \forall x A} & \begin{array}{l}
y \notin F V(\Gamma \cup\{\forall x A\}) \\
\text { (Universal Generalization) }
\end{array} \\
\text { MR1 } \frac{\Gamma, B \vdash A \quad \Gamma, C \vdash A}{\Gamma, B \vee C \vdash A} & \text { (Reasoning by Cases) } \\
\text { MR2 } \frac{\Gamma, B(x / y) \vdash A}{\Gamma, \exists x B \vdash A} & \begin{array}{l}
y \notin F V(\Gamma \cup\{\exists x B, A\}) \\
\text { (Existential Instantiation) }
\end{array}
\end{array}
$$

To set them apart from the ordinary rules, RQ, MR1 and MR2 are often called meta-rules. These meta-rules are, properly understood, existential statements about the provability relation; for instance, MR1 is the existential statement that if there is a proof of $A$ from $\Gamma \cup\{B\}$ and a proof of $A$ from $\Gamma \cup\{C\}$, then there is a proof of $A$ from $\Gamma \cup\{B \vee C\}$. These two meta-rules are derivable in some logics, but are generally not derivable in logics which lack a deduction theorem such as $\bar{\nabla} \mathbf{K}_{3}^{d}$. The following inductive definition

\footnotetext{
${ }^{2}$ The $^{d}$ indicates that the meta-rules MR1 and MR2 are present. A two-way rule $A \dashv B$ is short for the rules $A \vdash B$ and $B \vdash A$.
}

Australasian Journal of Logic (14:1) 2017, Article no. 7 
of what a proof is is designed to make these meta-rules come out as true statements regarding the defined provability relation.

Definition 2. A proof of $A$ from $\Gamma$ in the logic $\boldsymbol{L}$ is a finite nested list of formulas $\left\langle\alpha_{1}, \ldots, \alpha_{n}\right\rangle$ such that $\alpha_{n}=A$ and every $\alpha_{i \leq n}$ is either

(1) a member of $\Gamma$

(2) a logical axiom

(3) there is a set $\Delta \subseteq\left\{\alpha_{j} \mid j<i\right\}$ such that $\Delta \vdash \alpha_{i}$ is an instance of a rule of $\boldsymbol{L}$

(4) there is a $j<i$ such that $\alpha_{j}$ is the formula $B(x / y)$, and $\alpha_{i}$ is the formula $\forall x B$ where $y \notin F V\left(\{\forall x B\} \cup\left(\Gamma \cap\left\{\alpha_{m} \mid m<j\right\}\right)\right)$

(5) there is a $j<i$ such that $\alpha_{j}$ is the formula $B \vee C$, and there is some $\alpha_{k<i}=\left\langle\beta_{k_{1}}, \beta_{k_{2}}\right\rangle$ where $\beta_{k_{1}}$ is a proof of $\alpha_{i}$ from $\Gamma \cup\{B\}$ in the logic $\boldsymbol{L}$ and $\beta_{k_{2}}$ is a proof of $\alpha_{i}$ from $\Gamma \cup\{C\}$ in the logic $\boldsymbol{L}$

(6) there is a $j<i$ such that $\alpha_{j}$ is the formula $\exists x B$, and some $\alpha_{k<i}=$ $\left\langle\beta_{1}, \ldots, \beta_{m}, \alpha_{i}\right\rangle$ is a proof of $\alpha_{i}$ from $\Gamma \cup\{B(x / y)\}$ in the logic $\boldsymbol{L}$ where $y \notin F V\left(\Gamma \cup\left\{\exists x B, \alpha_{i}\right\}\right)$.

The existential claim that there is such a proof is written $\Gamma \vdash_{\mathbf{L}} A .^{3}$

It will later be convenient to work with intersubstitutivity rules. Such rules will be on the form $\Gamma, \theta_{A} \vdash \theta_{B}$ and $\Gamma \vdash \theta_{A} \star \theta_{B}$, where $\star$ is some connective, and where $\theta_{B}$ is obtained from $\theta_{A}$ by substituting zero or more instances of the subformula $A$ in $\theta$ by $B$. As an instance of such a rule we have that $\forall \bar{x}(A \leftrightarrow B) \vdash \theta_{A} \leftrightarrow \theta_{B}$ is a derived rule in any relevant logic.

Definition 3. (Semantics of $\bar{\nabla} \mathbf{K}_{3}^{d}$ ) The semantics of $\bar{\nabla} \mathbf{K}_{3}^{d}$ is as follows: a model $\mathfrak{A}$ with quantification domain $|\mathfrak{A}|$ and variable assignment function $\mathfrak{s}$ interprets variables, names and function symbols in the familiar way; the model $\mathfrak{A}_{\mathfrak{s}}$ induces a term assignment function $\hat{\mathfrak{s}}:$ TERM $\mapsto|\mathfrak{A}|$ such that

- if $t$ is a variable, then $\hat{\mathfrak{s}}(t)=\mathfrak{s}(t)$

- if $t$ is a constant symbol, then $\hat{\mathfrak{s}}(t)=t^{\mathfrak{2}}$

- if $t$ is a function term $f\left(t_{1}, \ldots, t_{n}\right)$, then $\hat{\mathfrak{s}}(t)=f^{\mathfrak{2}}\left(\hat{\mathfrak{s}}\left(t_{1}\right), \ldots, \hat{\mathfrak{s}}\left(t_{n}\right)\right)$.

The valuespace of any model is $\{\perp, \mathbf{n}, \top\}$. If $A(\bar{x})$ is a n-ary atomic predicate, then $\mathfrak{A}(A)$ is a function from $|\mathfrak{A}|^{n}$ to $\{\perp, \mathbf{n}, \top\}$, and $\mathfrak{A}_{\mathfrak{s}}(A(\bar{x} / \bar{t})):=\mathfrak{H}(A)(\hat{\mathfrak{s}}(\bar{t}))$. The identity predicate, $\doteq$, is treated as a logical relation:

$$
\mathfrak{U}(\doteq)(a, b)=\top \Longleftrightarrow a=b \text { for any } a, b \in|\mathfrak{A}| \text {. }
$$

$\mathfrak{A}_{\mathfrak{s}}$ interprets the other logical vocabulary compositionally so that $\wedge$ and $\forall$ are interpreted as infimum over, and $\vee$ and $\exists$ as supremum over the following three-valued Kleene algebra and $\neg$ is interpreted according to the

${ }^{3}$ I will often drop the subscript $\mathbf{L}$ on the derivability relation. An example of the use of clauses (5) and (6) can be found in the proof of Lem. 16. I will refer to these clauses as MR1 and MR2. 
displayed matrix:

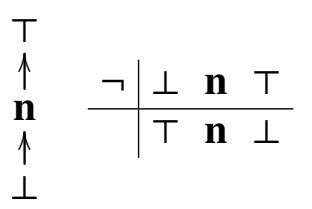

TRUTH IN A MODEL is defined in the standard way: $\mathfrak{A}_{\mathfrak{s}} \vDash A:=\mathfrak{A}_{\mathfrak{s}}(A)=\mathrm{T}$, and SEMANTIC CONSEQUENCE, is then defined as preservation of truth in all models: $\Theta \vDash A:=$ for all models $\mathfrak{A}_{\mathfrak{s}}$, if $\mathfrak{A}_{\mathfrak{s}} \vDash \theta$ for all $\theta \in \Theta$, then $\mathfrak{A}_{\mathfrak{s}} \vDash A$.

From the definition above it is easy to see that $\mathfrak{A}_{\mathfrak{s}} \vDash s \doteq t \Leftrightarrow \hat{\mathfrak{s}}(s)=\hat{\mathfrak{s}}(t)$, and so if $\mathfrak{A}_{\mathfrak{s}} \vDash s \doteq t$, then a simple induction on the complexity of $A$ will show that

$$
\mathfrak{A}_{\mathfrak{s}}(A(x / s, \bar{y}))=\mathfrak{A}(A)(\hat{\mathfrak{s}}(s), \hat{\mathfrak{s}}(\bar{y}))=\mathfrak{A}(A)(\hat{\mathfrak{s}}(t), \hat{\mathfrak{s}}(\bar{y}))=\mathfrak{A}_{\mathfrak{s}}(A(x / t, \bar{y}))
$$

where $A(x, \bar{y})$ is any formula and $x$ is any variable for which $s$ and $t$ are substitutable. Notice, however, that if $\hat{\mathfrak{s}}(s) \neq \hat{\mathfrak{s}}(t)$, then $\mathfrak{H}_{\mathfrak{s}}(s \doteq t)$ could be evaluated to either $\perp$ or $\mathbf{n}$.

E2 only ensures that an identity statement $s \doteq t$ comes out true if and only if $s$ and $t$ denote the same object and is for this reason a very weak version of Leibniz's law. For instance, it does not suffice for validating

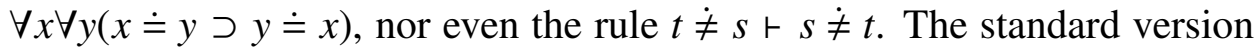
of Leibniz's law, $\forall x \forall y(x \doteq y \supset(A \supset A(x / y)))$, entails excluded middle for all formulas, and so collapses $\bar{\nabla} \mathbf{K}_{3}^{d}$ into classical logic. Adding E3 to $\bar{\nabla} \mathbf{K}_{3}^{d}$ does not suffices for this, however. That E3 does not entail excluded middle for all formulas is easily seen by noting that any two-element model can assign $A(x / t)$ the value $\mathrm{T}, t \doteq s$ the value $\perp$ and $A(x / s) \mathbf{n}$ as long as $A$ is some non-logical predicate. However, E3 does entail excluded middle for $\doteq$ :

Lemma 1. $\vdash_{\bar{\nabla} \mathbf{K}_{3}^{d}[E 3]} \forall x \forall y(x \not \dot{y} y \vee x \doteq y)$

Proof.
(1) $\forall x(x \doteq y / x)$
E1
(2) $x \doteq y / x$
1, Q1
(3) $\forall y(x \doteq y \supset x \doteq y) \quad 2, \mathrm{E} 3$
(4) $\forall x \forall y(x \neq y \vee x \doteq y) \quad 3$, RQ \& def. of $\supset$

Definition 4. (Semantics of $\left.\bar{\nabla} \mathbf{K}_{3}^{d}[E 3]\right)$ A model $\mathfrak{A}$ is a model for $\bar{\nabla} \mathbf{K}_{3}^{d}[E 3]$ if it is a model for $\bar{\nabla} \mathbf{K}_{3}^{d}$ such that $\mathfrak{A}(\dot{=})(a, b) \in\{\perp, \top\}$ for all $a, b \in|\mathfrak{A}|$.

E3 also entails the rule

$$
\text { E4 } A(x / t), \neg A(x / s) \vdash t \not \neq s \quad s \& t \text { free for } x
$$

since modus tollens is a valid rule in $\bar{\nabla} \mathbf{K}_{3}^{d}$. One could consider adding E4 as a primitive rule to $\bar{\nabla} \mathbf{K}_{3}^{d}$, but I have found it difficult to find a suitable Australasian Journal of Logic (14:1) 2017, Article no. 7 
semantic clause which would ensure a sound and complete semantics for $\bar{\nabla} \mathbf{K}_{3}^{d}[E 4]$ and so will only consider E4 as a derived rule of $\bar{\nabla} \mathbf{K}_{3}^{d}[E 3]$ when I need to appeal to semantic facts.

From the definition of truth in a model, together with the matrix for $\neg$, it follows that $\bar{\nabla} \mathbf{K}_{3}^{d}$ regards both $\mathbf{n}$ and $\neg \mathbf{n}$ as undesignated; if a sentence $A$ is evaluated to $\mathbf{n}$ in a model $\mathfrak{A}_{\mathfrak{s}}$, then neither $A$ nor $\neg A$ are true in $\mathfrak{A}_{\mathfrak{s}} . \bar{\nabla} \mathbf{K}_{3}^{d}$ is for this reason called a paracomplete logic.

Graham Priest introduced in [16] a logic which he called Logic of Paradox, $\mathbf{L P}$ for short. The first-order version of this logic with identity is quite closely related to $\bar{\nabla} \mathbf{K}_{3}^{d}$, but whereas $\bar{\nabla} \mathbf{K}_{3}^{d}$ regards $\mathbf{n}$ as undesignated, $\bar{\nabla} \mathbf{L} \mathbf{P}^{d}$ regards it as designated, so if a sentence $A$ is evaluated to it in a model $\mathfrak{A}_{\mathfrak{s}}$, then both $A$ and $\neg A$ are true in $\mathfrak{A}_{\mathfrak{s}} . \quad \bar{\nabla} \mathbf{L} \mathbf{P}^{d}$ is for this reason called a paraconsistent logic.

Proof-theoretically, $\bar{\nabla} \mathbf{L} \mathbf{P}^{d}$ is got from $\bar{\nabla} \mathbf{K}_{3}^{d}$ by dropping disjunctive syllogism (R7), and adding excluded middle, $A \vee \neg A$.

Definition 5. (Semantics of $\bar{\nabla} \mathbf{L} \mathbf{P}^{d}$ ) A $\bar{\nabla} \mathbf{L P}^{d}$-model $\mathfrak{A}_{\mathfrak{s}}$ is a $\bar{\nabla} \mathbf{K}_{3}^{d}$-model in

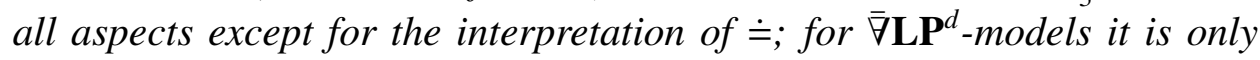
demanded that

$$
\mathfrak{A}(\doteq)(a, b) \in\{\mathbf{n}, \top\} \Longleftrightarrow a=b \text { for any } a, b \in|\mathfrak{A}| \text {. }
$$

TRUTH IN A MODEL $i$ defined in the standard way: $\mathfrak{U}_{\mathfrak{s}} \vDash A:=\mathfrak{A}_{\mathfrak{s}}(A) \in\{\mathbf{n}, \top\}$, and SEMANTIC CONSEQUENCE is then defined as preservation of truth in all models: $\Theta \vDash A:=$ for all models $\mathfrak{A}_{\mathfrak{s}}$, if $\mathfrak{A}_{\mathfrak{s}} \vDash \theta$ for all $\theta \in \Theta$, then $\mathfrak{A}_{\mathfrak{s}} \vDash A$.

For consistency with standard notation for $\bar{\nabla} \mathbf{L} \mathbf{P}^{d}$-models, I'll use ' $\mathbf{b}$ ' instead of 'n' when $\mathbf{b}$ is a truth-value of a $\bar{\nabla} \mathbf{L} \mathbf{P}^{d}$-model, and 'n' when it is a truth-value of a $\bar{\nabla} \mathbf{K}_{3}^{d}$-model.

Definition 6. Let $\leq$ be the following ordering:

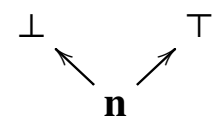

Let $\mathfrak{A}$ and $\mathfrak{B}$ be two models for $\bar{\nabla} \mathbf{K}_{3}^{d}\left(\bar{\nabla} \mathbf{L} \mathbf{P}^{d}\right)$. $\mathfrak{A} \leqslant \mathfrak{B}:=|\mathfrak{A}|=|\mathfrak{B}|$, $\mathfrak{A}$ and $\mathfrak{B}$ are identical with regards to how they interpret names and function symbols, and for every atomic predicate $F$, and every variable assignment function $\mathfrak{s ,} \mathfrak{A}_{\mathfrak{s}}(F) \leq \mathfrak{B}_{\mathfrak{s}}(F)$.

Theorem 2. (Monotonicity) $\bar{\nabla} \mathbf{K}_{3}^{d}, \bar{\nabla} \mathbf{K}_{3}^{d}[E 3]$ and $\bar{\nabla} \mathbf{L} \mathbf{P}^{d}$ are monotonic with regards to the ordering $\leq$; for any formula $A$ and models $\mathfrak{A}$ and $\mathfrak{B}$, if $\mathfrak{A} \leqslant \mathfrak{B}$, then $\mathfrak{A}_{\mathfrak{s}}(A) \leq \mathfrak{B}_{\mathfrak{s}}(A)$ for every variable assignment function $\mathfrak{s}$.

Proof. An easy induction on the complexity of $A$. 
Theorem 3. For any model $\mathfrak{A}$ and formulas $D$ and $E$, if $\mathfrak{A}_{\mathfrak{s}}(D) \leq \mathfrak{A}_{\mathfrak{5}}(E)$ for every variable assignment function $\mathfrak{s}$, then $\mathfrak{A}_{\mathfrak{s}}\left(\psi_{D}\right) \leq \mathfrak{A}_{\mathfrak{s}}\left(\psi_{E}\right)$ for every variable assignment function $\mathfrak{s}$.

Proof. Assume that $\mathfrak{H}_{\mathfrak{5}}(D) \leq \mathfrak{U}_{\mathfrak{s}}(E)$ for every variable assignment function s. Now add a new atomic predicate $F(\bar{x})$ such that $\bar{x}$ are the free variables in $D$ and $E$. Let $\mathfrak{H}^{\prime}$ be identical to $\mathfrak{A}$, but let it interpret the new predicate $F$ such that $\mathfrak{U}_{\mathfrak{s}}^{\prime}(F(\bar{x}))=\mathfrak{A}_{\mathfrak{s}}(D)$ for every variable assignment function $\mathfrak{s}$. Similarly, let $\mathfrak{U}_{\mathfrak{s}}^{\prime \prime}(F(\bar{x}))=\mathfrak{U}_{\mathfrak{s}}(E)$ for every variable assignment function $\mathfrak{s}$. Then $\mathfrak{\mathfrak { U } ^ { \prime }} \leqslant \mathfrak{U}^{\prime \prime}$ and so it follows from Thm. 2 that $\mathfrak{U}_{\mathfrak{s}}^{\prime}\left(\psi_{F(\bar{x})}\right) \leq \mathfrak{A}_{\mathfrak{s}}^{\prime \prime}\left(\psi_{F(\bar{x})}\right)$. By an easy induction on the complexity of $\psi$, it follows that $\mathfrak{A}_{\mathfrak{s}}\left(\psi_{D}\right)=\mathfrak{H}_{\mathfrak{s}}^{\prime}\left(\psi_{F(\bar{x})}\right)$ and $\mathfrak{A}_{\mathfrak{s}}\left(\psi_{E}\right)=\mathfrak{U}_{\mathfrak{s}}^{\prime \prime}\left(\psi_{F(\bar{x})}\right)$ which suffices for showing that $\mathfrak{U}_{\mathfrak{s}}\left(\psi_{D}\right) \leq \mathfrak{A}_{\mathfrak{s}}\left(\psi_{E}\right)$.

Corollary 4. For any model $\mathfrak{A}$ and formulas $D$ and $E$, if $\mathfrak{A}_{\mathfrak{s}}(D)=\mathfrak{A}_{\mathfrak{s}}(E)$ for every variable assignment function $\mathfrak{s}$, then $\mathfrak{A}_{\mathfrak{s}}\left(\psi_{D}\right)=\mathfrak{A}_{\mathfrak{s}}\left(\psi_{E}\right)$ for every variable assignment function $\mathfrak{s}$.

Proof. Immediate from Thm. 3

Definition 7. Let $\Delta$ and $\Delta^{\prime}$ be sets of formulas over, respectively, the languages $\mathcal{L}$ and $\mathcal{L}^{\prime}$ where $\mathcal{L}^{\prime}$ extends $\mathcal{L}$. $\Delta^{\prime}$ CONSERvatively EXTENDs $\Delta$ in the $\operatorname{logic} \mathbf{L}$ if for every formula $A$ in the language $\mathcal{L}$,

$$
\Delta^{\prime} \vdash_{\mathbf{L}} A \Rightarrow \Delta \vdash_{\mathbf{L}} A \text {. }
$$

Definition 8. A logic $\mathbf{L}$ is Conservatively extended by a rule $\Theta \vdash A$ if $\Delta^{\prime}$ conservatively extends $\Delta$ where $\Delta$ is any set offormulas and $\Delta^{\prime}$ is the closure of $\Delta$ with respect to $\mathbf{L}$ and the rule $\Theta \vdash A$. Derivatively: if $R$ is a rule, then the logic $\mathbf{L}[R]-\mathbf{L}$ strengthened with $R$-conservatively extends $\mathbf{L}$ if $\mathbf{L}$ is conservatively extended by $R$.

Since I will consider rules which extend the language it is important to note that I will assume that if $\Theta \vdash A$ is a rule of a $\operatorname{logic} \mathbf{L}$ which is augmented with a rule $R$, then one is entitled to infer $B$ from $\Psi$ in $\mathbf{L}[R]$ if $\Psi \vdash B$ is an instance of the rule $\Theta \vdash A$, but with formulas over the augmented language which $R$ introduced.

\section{Henkin constants}

This section shows how to conservatively add Henkin constants. Constants are simply 0 -ary functions and so Henkin constants are simply 0 -ary Skolem functions. The next section shows how to add $n$-ary Skolem functions.

Definition 9. Let $\Theta$ be a set of formulas in the language $\mathcal{L}$. Let hc $(\mathcal{L})$ be the language $\mathcal{L}$ extended by adding a HeNKIn CONSTANT $c_{B}$ for every formula

Australasian Journal of Logic (14:1) 2017, Article no. 7 
$\exists x B$ in the language of $\mathcal{L}$.

$$
\Theta_{c}:=\left\{B\left(x / c_{B}\right) \mid \Theta \vdash \exists x B\right\}
$$

$\Theta^{h c}:=\Theta_{c} \cup \Theta$ is called the Henkin-witnessed extension of $\Theta . B\left(x / c_{B}\right)$ is called $a$ Henkin witness. ${ }^{4}$

Theorem 5. $\Theta^{h c}$ conservatively extends $\Theta$.

Proof. Let $\Theta \cup\{A\}$ be a set of formulas over the language $\mathcal{L}$ and assume that $\Theta^{h c}+A$. Since proofs are finite we have that for some finite subsets $\Theta^{\prime} \subseteq \Theta$ and $\left\{B_{1}\left(c_{B_{1}}\right), \ldots, B_{n}\left(c_{B_{n}}\right)\right\} \subseteq \Theta_{c}$,

$$
\left\{B_{1}\left(c_{B_{1}}\right), \ldots, B_{n-1}\left(c_{B_{n-1}}\right)\right\} \cup \Theta^{\prime}, B_{n}\left(c_{B_{n}}\right) \vdash A,
$$

where for each $B_{i}, \Theta \vdash \exists x B_{i}$. Since the Henkin constant $c_{B_{n}}$ does not occur in $\left\{B_{1}\left(c_{B_{1}}\right), \ldots, B_{n-1}\left(c_{B_{n-1}}\right)\right\} \cup \Theta^{\prime} \cup\{A\}$ we can simply replace $c_{B_{n}}$ by a variable $y$ which does not occur in $\left\{B_{1}\left(c_{B_{1}}\right), \ldots, B_{n-1}\left(c_{B_{n-1}}\right)\right\} \cup \Theta^{\prime} \cup\{A\}$ so that

$$
\left\{B_{1}\left(c_{B_{1}}\right), \ldots, B_{n-1}\left(c_{B_{n-1}}\right)\right\} \cup \Theta^{\prime}, B_{n}(x / y) \vdash A .
$$

Using MR2 we can now infer that

$$
\left\{B_{1}\left(c_{B_{1}}\right), \ldots, B_{n-1}\left(c_{B_{n-1}}\right)\right\} \cup \Theta^{\prime}, \exists x B_{n} \vdash A .
$$

Repeating the procedure we get that $\left\{\exists x B_{1}, \ldots, \exists x B_{n}\right\} \cup \Theta^{\prime} \vdash A$. Since $\Theta \vdash$ $\exists x B_{i}$ and $\Theta^{\prime} \subseteq \Theta$, we have that $\Theta \vdash A$.

Note that the only assumption used regarding the logic in Thm. 5 was that it validates the meta-rule MR2. In fact the converse is also the case:

Theorem 6. If $\Theta^{h c}$ conservatively extends $\Theta$, then $\Theta$ is closed under MR2.

Proof. Assume that $\Theta, A(x / y) \vdash B$, where $y \notin F V(\Theta \cup\{\exists x A, B\})$, that $\Theta \vdash$ $\exists x A$, and that $\Theta^{h c}$ conservatively extends $\Theta$. The goal is showing that $\Theta \vdash B$. Now since $\Theta \vdash \exists x A$, we get that $\Theta^{h c} \vdash A\left(x / c_{A}\right)$, where $c_{A}$ is the Henkin constant for $A$. Since $\Theta, A(x / y) \vdash B$ where $y \notin F V(\Theta \cup\{\exists x A, B\})$ we can rewrite the proof using $c_{A}$ instead of $y$ so that $\Theta, A\left(x / c_{A}\right)+B$. By the construction of $\Theta^{h c}$ we get that $\Theta^{h c} \vdash A\left(x / c_{A}\right)$ and so by transitivity of $\vdash$ that $\Theta^{h c} \vdash B$. Since $B$ is a formula in the language of $\Theta$ and $\Theta^{h c}$ by assumption conservatively extends $\Theta$, we can conclude that $\Theta \vdash B$.

Definition 10. For any language $\mathcal{L}$, let $\mathcal{L}_{h_{0}}:=\mathcal{L}, \mathcal{L}_{h_{n+1}}:=h c\left(\mathcal{L}_{h_{n}}\right)$ and $\mathcal{L}_{h_{\omega}}:=$ $\bigcup_{i<\omega} h c\left(\mathcal{L}_{h_{i}}\right)$. Furthermore let

\footnotetext{
${ }^{4}$ It would be more appropriate to use $\exists x B$ instead of $B$ as a subscript in $c_{B}$ - to let $c_{\exists x B}$ be the Henkin constant for the formula $\exists x B$. I trust that no confusion will arise and so I'll stick to the neater, although flawed, naming convention.
}

Australasian Journal of Logic (14:1) 2017, Article no. 7 


$$
\begin{aligned}
& \Theta^{h c_{0}}:=\Theta \\
& \Theta^{h c_{n+1}}:=\Theta^{h c_{n}} \cup\left\{B\left(y / c_{B}\right) \mid \Theta^{h c_{n}}+\exists y B\right\} \\
& \Theta^{h c_{\omega}}:=\bigcup_{i<\omega} \Theta^{h c_{i}}
\end{aligned}
$$

Theorem 7. $\Theta^{h c_{\omega}}$ conservatively extends $\Theta$.

Proof. The above theorem shows that $\Theta^{h c_{0}}$ is conservatively extended by $\Theta^{h c_{1}}$. It is easy to see that the proof holds for any $n$ - that $\Theta^{h c_{n}}$ is conservatively extended by $\Theta^{h c_{n+1}}$. Furthermore, it is obvious that if $\Delta_{1}$ is conservatively extended by $\Delta_{2}$ and $\Delta_{2}$ is conservatively extended by $\Delta_{3}$, then $\Delta_{1}$ is conservatively extended by $\Delta_{3}$, and so $\Theta$ is conservatively extended by every $\Theta^{h c_{n}}$. Now since deductions are finite it follows from the assumption that $\Theta^{h c_{\omega}} \vdash A$ for $A \in \mathcal{L}$, that for some $m, \Theta^{h c_{m}} \vdash A$. Since $\Theta$ is conservatively extended by $\Theta^{h c_{m}}$ and $A \in \mathcal{L}$ it follows that $\Theta \vdash A$.

\section{Definition 11.}

$$
\text { (Henkin rule) } \exists y A(y) \vdash A\left(y / c_{A}\right)
$$

where if $\exists y A(y)$ is a formula of $\mathcal{L}_{h_{n}}, A\left(y / c_{A}\right)$ is a formula of $\mathcal{L}_{h_{n+1}}$.

Corollary 8. Adding the Henkin rule yields a conservative extension.

Proof. This follows from Thm. 7.

This section has shown that one can add Henkin constants conservatively. Constants are simply 0 -ary functions. The next section shows how to extend the results in this section so as to cover $n$-ary functions for arbitrary $n$.

The results in the next section depends on the strong completeness theorem which says that if $A$ holds in every model in which $\Sigma$ does, then $A$ is derivable from $\Sigma$, or, equivalently, if $A$ is not derivable from $\Sigma$, then there is a model in which $\Sigma$ holds, but $A$ fails $-\Sigma \nvdash A \Rightarrow \Sigma \not A$. Henkin proved this theorem in [10] by showing that it is possible to extend $\Sigma$ into a set of formulas $\Gamma$ and construct from it a model for $\Sigma$ in which $A$ fails. In order to validate the meta-rule MR2 a model needs to be witnessed-if $\mathfrak{A}_{\mathfrak{s}} \vDash \exists x B$, then $\mathfrak{A}_{\mathfrak{s}(x / a)} \vDash B$ for some object $a \in|\mathfrak{x}|$. Adding Henkin constants ensures that the model is witnessed and the conservativeness property ensures that such an extension retains the property of keeping $A$ non-derivable.

Fact 1. $\bar{\nabla} \mathbf{K}_{3}^{d}, \bar{\nabla} \mathbf{K}_{3}^{d}[E 3]$ and $\bar{\nabla} \mathbf{L P}^{d}$ are strongly sound and complete with regards to the semantics defined above:

$$
\begin{aligned}
\Theta \vDash_{\bar{\nabla} \mathbf{K}_{3}^{d}} A & \Longleftrightarrow \Theta \vdash_{\bar{\nabla} \mathbf{K}_{3}^{d}} A \\
\Theta \vDash_{\bar{\nabla} \mathbf{K}_{3}^{d}[E 3]} A & \Longleftrightarrow \Theta \vdash_{\bar{\nabla} \mathbf{K}_{3}^{d}[E 3]} A \\
\Theta \vDash_{\bar{\nabla} \mathbf{L} \mathbf{P}^{d}} A & \Longleftrightarrow \Theta \vdash_{\bar{\nabla} \mathbf{L} \mathbf{P}^{d}} A
\end{aligned}
$$

Australasian Journal of Logic (14:1) 2017, Article no. 7 
This is a substantial result. Proving it, however, would make this paper unnecessarily long, and so I will have to defer it to some other occasion. Soundness and completeness proofs for variants of the logics $\bar{\nabla} \mathbf{K}_{3}^{d}$ and $\bar{\nabla} \mathbf{L} \mathbf{P}^{d}$ can be found in Priest's book [17, ch. 22]. Beyond relying on the meta-rule MR2 and the fact that any model interprets compositionally, that is the semantic value assigned to a term or formula depends only on the semantic values of its subterms/subformulas, the results of the next section, like the results in this section, will not otherwise depend on any particular rule of $\bar{\nabla} \mathbf{K}_{3}^{d}$, and so will hold for other logics with a similary sound and complete algebraic semantics such as $\bar{\nabla} \mathbf{K}_{3}^{d}[E 3]$ and $\bar{\nabla} \mathbf{L} \mathbf{P}^{d}$, the four-valued logic $\bar{\nabla} \mathbf{F D E}{ }^{d}$, the $n$-valued logics $\bar{\nabla} \mathbf{R} \mathbf{M}_{n}^{d}$ and Lukasiewicz logics, and intensional logics such as intuitionistic logic, relevant logics and the infinitevalued Łukasiewicz logic. There is a caveat, however: if the logic has any other version of Leibniz's law than E2, then the results may not hold.

\section{SKolem FUnctions}

This section extendeds the results of the previous section by showing how to conservatively add Skolem functions.

Definition 12. Let $\Theta$ be a set of formulas in the language $\mathcal{L}$. Let $s f(\mathcal{L})$ be the language $\mathcal{L}$ extended by adding a Skolem function $f_{B}(\bar{x})$ for every formula $B(\bar{x}, y)$ in the language of $\mathcal{L}$.

$$
\Theta_{f}:=\left\{\forall \bar{x} B\left(y / f_{B}(\bar{x})\right) \mid \Theta \vdash \forall \bar{x} \exists y B\right\}
$$

$\Theta^{s f}:=\Theta_{f} \cup \Theta$ is called the Skolem extension of $\Theta$.

Theorem 9. $\Theta^{s f}$ conservatively extends $\Theta$

Proof. Assume that $\Theta \nvdash A$. Using the completeness theorem we can infer that $\Theta \not z A$. Let $\mathfrak{A}_{\mathfrak{s}}$ be any model such that $\mathfrak{A}_{\mathfrak{s}} \vDash \Theta$ and $\mathfrak{A}_{\mathfrak{s}} \not \models A$. For every formula $\forall \bar{x} B\left(y / f_{B}(\bar{x})\right) \in \Theta_{f}$ we have that $\mathfrak{A}_{\mathfrak{s}} \vDash \forall \bar{x} \exists y B(\bar{x}, y)$, and so $\mathfrak{A}_{\mathfrak{s}(\bar{x} / \bar{a})} \vDash$ $\exists y B(\bar{x}, y)$ for every $\bar{a} \in|\mathfrak{R}|^{n}$. Since every model is witnessed it follows that $W_{\bar{a}}^{B(\bar{x}, y)}:=\left\{b \in|\mathfrak{A}| \mid \mathfrak{A}_{\mathfrak{s}(\bar{x} / \bar{a}, y / b)} \vDash B(\bar{x}, y)\right\}$ is non-empty for each $\bar{a} \in|\mathfrak{A}|^{n}$. Let the axiom of choice choose one such element for every $\bar{a} \in|\mathfrak{A}|^{n}$ and use it as the denotation of the Skolem function $f_{B}(\bar{x})$. Let $\mathfrak{U}^{s f}$ be the model which results from giving such interpretations to each Skolem function in $\Theta_{f}$. It then follows that $\mathfrak{I}_{\mathfrak{s}}^{s f} \vDash \Theta_{f}$. Since $\mathfrak{A}_{\mathfrak{s}}^{s f_{\omega}}$ is compositional it follows that it assigns the same values to any term and formula in $\mathcal{L}$, and therefore that $\mathfrak{U}_{\mathfrak{s}}^{s f_{\omega}} \not \models A$. The soundness theorem then ensures that $\Theta^{s f} \nvdash A$.

If $\Theta \vdash \forall x \exists y \exists z A(x, y, z)$, then $\Theta^{s f} \vdash \forall x \exists z A(x, f(x), z)$ for some Skolem function $f$. To get rid of the last existential quantifier we need to repeat the process: $\left(\Theta^{s f}\right)^{s f} \vdash \forall x A(x, f(x), g(x))$ for some Skolem function $g$. The next 
definition and theorem makes this precise and shows how to add Skolem functions an masse.

Definition 13. For any language $\mathcal{L}$, let $\mathcal{L}_{s_{0}}:=\mathcal{L}, \mathcal{L}_{s_{n+1}}:=s f\left(\mathcal{L}_{s_{n}}\right)$ and $\mathcal{L}_{s_{\omega}}:=$ $\bigcup_{i<\omega} s f\left(\mathcal{L}_{s_{i}}\right)$. Furthermore let

$$
\begin{aligned}
& \Theta^{s f_{0}}:=\Theta \\
& \Theta^{s f_{n+1}}:=\Theta^{s f_{n}} \cup\left\{\forall \bar{x} B\left(y / f_{B}(\bar{x})\right) \mid \Theta^{s f_{n}} \vdash \forall \bar{x} \exists y B\right\} \\
& \Theta^{s f_{\omega}}:=\bigcup_{i<\omega} \Theta_{i}^{s f}
\end{aligned}
$$

Theorem 10. $\Theta^{s f_{\omega}}$ conservatively extends $\Theta$.

Proof. Similar to the proof of Thm. 7.

\section{Definition 14.}

$($ Skolem rule; $\mathcal{S}) \quad \forall \bar{x} \exists y A(\bar{x}, y) \vdash \forall \bar{x} A\left(\bar{x}, y / f_{A}(\bar{x})\right)$

where if $A(\bar{x}, y)$ is a formula of $\mathcal{L}_{S_{n}}, A\left(\bar{x}, y / f_{A}(\bar{x})\right)$ is a formula of $\mathcal{L}_{S_{n+1}}$.

\section{Lemma 11.}

$$
\Theta \vdash_{\bar{\nabla} \mathbf{K}_{3}^{d}[\mathcal{S}]} A \Longleftrightarrow \Theta^{s f_{\omega}} \vdash_{\bar{\nabla} \mathbf{K}_{3}^{d}} A
$$

Proof. Trivially by the definition of $\Theta^{s f_{\omega}}$.

Corollary 12. $\bar{\nabla} \mathbf{K}_{3}^{d}[\mathcal{S}]$ conservatively extends $\bar{\nabla} \mathbf{K}_{3}^{d}$.

Proof. This follows from Thm. 10 together with Lem. 11.

That the Skolem extension of any model validates E2 is quite trivial; if the extended model validates $t \doteq s$ for $t, s \in \mathcal{L}_{s_{\omega}}$, then it evaluates these two terms to the same element, and so the value assigned to $A(x / t)$ will have to be the same value as that assigned to $A(x / s)$ (assuming that $t$ and $s$ are both free for $x$ in $A$ ). Note, however, that this may not be the case for other versions of Leibniz's law than E2. It does, however, hold for E3:

Lemma 13. For any $\bar{\nabla} \mathbf{K}_{3}^{d}$-model $\mathfrak{A}_{\mathfrak{s}}$, if $\mathfrak{A}_{\mathfrak{s}}$ validates E3, then any Skolem extension of $\mathfrak{A}_{\mathfrak{s}}$ got from $\mathfrak{A}_{\mathfrak{s}}$ using the construction in Thm. 9 validates E3 over the full language $\mathcal{L}_{s_{\omega}}$.

Proof. Let $A$ be any formula and $t$ any term free for $x$ such that $\mathfrak{U}_{\mathfrak{s}}^{s f_{\omega}}(A(x / t))=$ T. By Lem. 1 it follows that for any element $a \in\left|\mathfrak{A}^{s f_{\omega}}\right|$, either $\mathfrak{A}_{\mathfrak{s}(x / a)}^{s f_{\omega}}(t \dot{=} x)=\top$ or $\mathfrak{I}_{\mathfrak{s}(x / a)}^{s f_{\omega}}(t \doteq x)=\perp$. If the latter holds, then obviously $\mathfrak{A}_{\mathfrak{s}(x / a)}^{s f_{\omega}}(t \doteq x \supset A)=\mathrm{T}$, and if the first holds, then $\mathfrak{U}_{\mathfrak{s}}^{s f_{\omega}}(A(x / t))=\mathfrak{U}_{\mathfrak{s}(x / a)}^{s f_{\omega}}(A)$ and so again $\mathfrak{H}_{\mathfrak{s}(x / a)}^{s f_{\omega}}(t \doteq x \supset A)=\mathrm{T}$. Since this holds for any $a \in\left|\mathfrak{I}^{s f_{\omega}}\right|$, it follows that $\mathfrak{H}_{\mathfrak{s}}^{s f_{\omega}}(\forall x(t \doteq x \supset A))=\mathrm{T}$.

Corollary 14. $\bar{\nabla} \mathbf{K}_{3}^{d}[\mathcal{S}, E 3]$ conservatively extends $\bar{\nabla} \mathbf{K}_{3}^{d}[E 3]$. Australasian Journal of Logic (14:1) 2017, Article no. 7 
Intuitionistic logic is often proclaimed as a logic in which one cannot add Skolem functions conservatively. That this is so goes back to Grigori Mints' paper [13]. The standard counterexample is due to Smoryński's paper [22] in which a countermodel for the formula $\forall x_{1} \exists y_{1} \forall x_{2} \exists y_{2}\left(x_{1} \not y_{1} \wedge\right.$ $\left.x_{2} \not y_{2} \wedge\left(x_{1} \doteq x_{2} \rightarrow y_{1} \doteq y_{2}\right)\right)$ is provided. This formula is easily derivable from $\forall x(x \neq f(x)) \wedge \forall x \forall y(x \doteq y \rightarrow f(x) \doteq f(y))$ where $f$ is introduced as a Skolem function for the formula $\forall x \exists y(x \neq y)$. A function $f$ is said to be extensional if $\forall x \forall y(x \doteq y \rightarrow f(x) \doteq f(y))$ holds. Smoryński's countermodel then shows that the addition of extensional Skolem functions is not always conservative. The crucial assumption here is undoubtedly the requirement that $f$ be extensional; intuitionistic logic with E1 and E2 and using the definition of a proof in this paper, can be shown to be sound and complete with regards to an algebraic semantics over Heyting algebras. The construction in Thm. 9 therefore also holds for this logic and so shows that Skolem functions can be added conservatively. The crucial assumption therefore is that of extensionality. ${ }^{5}$ Note that the same also holds for $\bar{\nabla} \mathbf{K}_{3}^{d}: \forall x_{1} \exists y_{1} \forall x_{2} \exists y_{2}\left(x_{1} \not y_{1} \wedge x_{2} \dot{\neq} y_{2} \wedge\left(x_{1} \doteq x_{2} \supset y_{1} \doteq y_{2}\right)\right)$ is derivable from $\forall x(x \not f(x)) \wedge \forall x \forall y(x \doteq y \supset f(x) \doteq f(y))$ where $f$ is introduced as a Skolem function for the formula $\forall x \exists y(x \neq y)$. Now let the language be the pure language of identity, $|\mathfrak{A}|=\{a, b, c\}$, and let $\mathfrak{A}$ interpret $\doteq$ according to the following matrix:

$$
\begin{array}{c|ccc}
\doteq & a & b & c \\
\hline a & \top & \mathbf{n} & \perp \\
b & \perp & \top & \mathbf{n} \\
c & \perp & \mathbf{n} & \top
\end{array}
$$

It is easy to verify that $\forall x_{1} \exists y_{1} \forall x_{2} \exists y_{2}\left(x_{1} \dot{\neq} y_{1} \wedge x_{2} \dot{\neq} y_{2} \wedge\left(x_{1} \doteq x_{2} \supset y_{1} \dot{=} y_{2}\right)\right)$ is evaluated to $\mathbf{n}$ in $\mathfrak{A}$ : when $x_{1}$ and $x_{2}$ are assigned to, respectively, $a$ and $b$, there is only one assignment to $y_{1}$ and $y_{2}$ which ensures that $x_{1} \neq y_{1} \wedge x_{2} \dot{\neq} y_{2}$ is true, namely $c$ and $a$. However, since $\mathfrak{A}(\dot{=})(a, b)=\mathbf{n}$ and $\mathfrak{A}(\dot{=})(c, a)=\perp$, it follows that $\mathfrak{A}\left(\forall x_{1} \exists y_{1} \forall x_{2} \exists y_{2}\left(x_{1} \dot{\neq} y_{1} \wedge x_{2} \dot{\neq} y_{2} \wedge\left(x_{1} \doteq x_{2} \supset y_{1} \doteq y_{2}\right)\right)\right)=\mathbf{n}$. We therefore have the following corollary:

\footnotetext{
${ }^{5}$ Note that intuitionistic logic is most often stated as a logic without identity; the identity predicate is then regarded as a non-logical predicate and one seeks to derive Leibniz's law from the axioms stated for $\doteq$ together with the axioms of the other predicates and function symbols. For instance, one adds not only $\forall x \forall y(s(x) \doteq s(y) \rightarrow x \doteq y)$ when stating that the successor function is injective in intuitionistic arithmetic, but also that it is extensional; $\forall x \forall y(x \doteq y \rightarrow s(x) \doteq s(y))$ is in other words added as a separate arithmetical axiom. Regardless of this, it is unnecessarily confusing to, as is often the case, claim that Skolem functions can't always be conservatively added to intuitionistic logic when intending to claim that extensional Skolem functions can't always be conservatively added.
} 
Corollary 15. $\bar{\forall} \mathbf{K}_{3}^{d}$ cannot be conservatively extended by $\supset$-extensional Skolem functions.

Note, however, that Skolem functions are derivably extensional in $\bar{\nabla} \mathbf{K}_{3}^{d}[E 3]$ which therefore can be conservatively extended by $\supset$-extensional Skolem functions.

Weber, in the second appendix of [24], raised the question whether it is possible to conservatively extend a logic by adding a rule governing Skolem functions. Cor. 12 settled this in the positive. ${ }^{6}$

The Skolem rule allows one to conservatively introduce a function symbol for all $n+1$-ary relations which relates all $n$-tuples of objects to at least one thing. The more interesting question is what kind of reasoning is warranted when one introduces function symbols for relations which provably relates all $n$-tuples to just one thing. Weber, in the above mentioned appendix, considered adding a rule for what he called unique objects, namely the rule

$$
\frac{\vdash \exists x \Phi(x) \quad \Phi(y) \vdash x \doteq y}{\vdash \Phi\left(f_{\Phi}\right)} .
$$

There are several problems with this rule as it stands. For present purposes it suffices to notice that the variable $x$ in $x \doteq y$ is free and not bound by the existential quantifier, and so the premises taken together can't plausibly be taken to express the claim that there exists one and only one $\Phi$. The trouble here is how to define unique existence. As I have not been able to come up with a variant of Weber's rule which essentially involves $\vdash$, and doubt that there is one, I will only consider the more standard ways of defining $\exists$ !.

The next sections looks at different ways of adding rules for reasoning with definable Skolem functions and functional formulas, that is a formula $A(\bar{x}, y)$ for which $\forall \bar{x} \exists ! y(A(\bar{x}, y))$ is derivable relative to some theory and definition of $\exists$ !.

\section{Definable Skolem functions}

Being able to introduce definitions when arguing is quite essential; in many cases it would be simply too hard to reason properly, or even articulate an idea if one were not allowed to use defined terms and relations. This section introduces definable Skolem functions, defines the unique existential quantifier, $\exists$ !, and introduces substitutivity rules relating $f_{A}(\bar{t}, s)$ to $A(\bar{t}, s)$. Before I start, let me note that introducing new predicates/terms for

\footnotetext{
${ }^{6}[24]$ contains a proof to the effect that the Skolem rule is conservative with regards to the truth-constant $\perp$. Weber's proof makes use of Skolem axioms instead of the Skolem rule and needs the rule called Independence of Premise in order for it to work. For more on this rule, see the appendix.
}

Australasian Journal of Logic (14:1) 2017, Article no. 7 
complex formulas/terms is easily seen to be conservative; if $A(\bar{x})$ is a logically complex formula and $f(\bar{y})$ is logically complex term, one can simply introduce a new predicate $B(\bar{x})$ and a new function symbol $g(\bar{y})$ and show, using the soundness and completeness theorem, that the introduction is conservative; simply assign the new vocabulary the same semantic value in any model as the semantic value of the formula or term they are intended to define.

I showed in the last section that the Skolem rule can be added conservatively to $\bar{\nabla} \mathbf{K}_{3}^{d}$. The Skolem rule in itself is close to useless; the only reasoning it by itself warrants is that given that some $n+1$-ary relation provably relates every $n$-tuple to some object, then one may introduce a function symbol which picks out one such object for each $n$-tuple. More precisely, it does not tell one how to eliminate a Skolem function symbol from a formula and it does not tell one anything about how $A(\bar{t}, s)$ and $s \doteq f_{A}(\bar{t})$ are related.

Definition 15. A formula $A(\bar{x}, y)$ is FUnctional in a logic $\mathbf{L}$ relative to $\Theta$ and a definition of $\exists$ ! if $\Theta \vdash_{\mathbf{L}} \forall \bar{x} \exists$ ! $y A(\bar{x}, y)$, and RELATIONAL if not.

Definition 16. A Skolem function is called DEFINABLE relative to some definition of $\exists$ ! if it is introduced for a functional formula.

Standard textbooks on classical mathematical logic usually prove the following two propositions regarding Skolem functions. ${ }^{7}$

Let $\Phi \cup\{A(\bar{x}, y)\}$ be any set of formulas over the language $\mathcal{L}$ such that

$$
\Phi \vdash \forall \bar{x} \exists ! y A(\bar{x}, y) .
$$

Let

$$
\begin{aligned}
\mathcal{L}^{+} & :=\mathcal{L} \cup\left\{f_{A}\right\} \\
\Phi^{+} & :=\Phi \cup\left\{\forall \bar{x} \forall y\left(A(\bar{x}, y) \leftrightarrow y \doteq f_{A}(\bar{x})\right\} .\right.
\end{aligned}
$$

(1) $\Phi^{+}$is a conservative extension of $\Phi$.

(2) There is a translation $*: \mathcal{L}^{+} \mapsto \mathcal{L}$ such that for any formula $B \in \mathcal{L}^{+}$,

$$
\Phi^{+} \vdash B \Longleftrightarrow \Phi \vdash B^{*} \text {. }
$$

The question now is whether these propositions also hold true of other logics than classical logic. There are two main challenges in simply stating these propositions for non-classical logics:

(i) How is the unique existential quantifier, $\exists$ !, to be defined?

(ii) Which axioms/rules are appropriate for relating a functional formula $A$ to the Skolem function $f_{A}$ ?

\footnotetext{
${ }^{7}$ See for instance [20, ch. 4.6] and [23, Thm. 3.4.6].
} 
Only by first answering these two questions can one hope to fruitfully answer whether adding such defining axioms or rules yields a conservative extension (1), and if there is a translation from the extended theory into the original one which preserves derivability (2).

To even get started I have decided upon answering (ii) first; if one is in some way able to prove that a formula $A(\bar{x}, y)$ determines the extension of a function - to prove $\forall \bar{x} \exists$ ! $y A(\bar{x}, y)$ for some definition of $\exists$ ! - then one would expect that $A(\bar{t}, s)$ and $s \doteq f_{A}(\bar{t})$ are intersubstitutable where $f_{A}$ is a Skolem function for $A$ and $\bar{t}$ and $s$ are any terms which are substitutable for $\bar{x}$ and $y$ in $A$. Although I will show that few logics validates this, I think that this is a rather unintuitive feature of these logics which ought to be stated clearly. The following definitions states the idea more precisely:

Definition 17. A logic $\mathbf{X}$ is Fit For SKolem functions Relative to A DEFiniTION OF $\exists$ ! if it can be conservatively extended by the Skolem rule together with the following two rules:

$$
\begin{array}{ll}
\text { (deSkolemizer) } & \forall \bar{x} \exists ! y A(\bar{x}, y), \psi_{s \doteq f_{A}(\bar{t})} \vdash \psi_{A(\bar{t}, s)} \\
\text { (Skolemizer) } & \forall \bar{x} \exists ! y A(\bar{x}, y), \psi_{A(\bar{t}, s)} \vdash \psi_{s=f_{A}(\bar{t})}
\end{array}
$$

where $\bar{t}$ and s are substitutable for $\bar{x}$ and $y$ in $A$, and $\psi_{A(\bar{t}, s)}$ is got from $\psi_{s \doteq f_{A}(\bar{t})}$ by replacing zero or more instances of $s \doteq f_{A}(\bar{t})$ in $\psi_{s \doteq f_{A}(\bar{t})}$ by $A(\bar{t}, s)$ and similarly for obtaining $\psi_{s \dot{=} f_{A}(\bar{t})}$ from $\psi_{A(\bar{t}, s)} .{ }^{8}$

Definition 18. A logic $\mathbf{L}$ is SEMIFIT for Skolem functions Relative to a DEFINITION of $\exists$ ! if it can be conservatively extended by the Skolem rule together with the deSkolemizer.

Definition 19. A logic $\mathbf{L}$ is Unfit For SKolem Functions Relative to A DEFINITION of $\exists$ ! if it is not semifit for Skolem functions relative to the definition of $\exists$ !.

«Parenthetical remark. Let me stress that the results on unfitness are always relative to some definition of the unique existential quantifier. I nowhere claim that results proven hold for every possible such definition, although I myself have not been able to come up with any other definitions which works better than those I mention. The challenge is therefore put to the defender of this or that logic to find a workable definition of the unique existential quantifier and to show that there are reasonable laws relating a Skolem equation $s \doteq f_{A}(\bar{t})$ to $A(\bar{t}, s)$. End parenthetical.»

The first challenge is to define $\exists$ ! - to find a workable way to express the quantifier phrase there exists one and only one $x$ such that. There are two standard ways of doing this:

${ }^{8}$ I will from now on assume that $\bar{t}$ and $s$ are substitutable for $\bar{x}$ and $y$ in $A$.

Australasian Journal of Logic (14:1) 2017, Article no. 7 


\section{Definition 20.}

$$
\begin{aligned}
& \exists ! x x A:=\exists x(A \wedge \forall z(A(x / z) \supset z \doteq x)) \\
& \exists ! \bar{x} A:=\exists x \forall z(A(x / z) \equiv z \doteq x) .
\end{aligned}
$$

These two definitions of $\exists$ ! are not equivalent in either $\bar{\forall} \mathbf{K}_{3}^{d}$ or $\bar{\forall} \mathbf{L} \mathbf{P}^{d}$ as

\begin{tabular}{|c|c|c|}
\hline & $\bar{\nabla} \mathbf{L} \mathbf{P}^{d}$ & $\bar{\nabla} \mathbf{K}_{3}^{d}$ \\
\hline$\forall \bar{x} \exists ! y A \vdash \forall \bar{x} \exists ! \overline{\bar{y}} A$ & $\checkmark$ & $x$ \\
\hline$\forall \bar{x} \exists ! \overline{\bar{y}} A \vdash \forall \bar{x} \exists ! \bar{y} A$ & $x$ & $\checkmark$ \\
\hline$\forall \bar{x} \exists ! y A \vdash \forall \bar{x} \forall y(\neg A \vee A)$ & $\checkmark$ & $\checkmark$ \\
\hline
\end{tabular}
Fig. 1 makes clear. ${ }^{9}$

FIGURE 1. Relations between two definitions of $\exists$ !

In order to make these two definitions of $\exists$ ! interderivable in $\bar{\nabla} \mathbf{L} \mathbf{P}^{d}$, one needs to add the rule $t \dot{\neq} t \vdash B$. I think most serious paraconsistentist would reject this rule, and so I will not discuss it further. In order to make these two definitions of $\exists$ ! interderivable in $\bar{\nabla} \mathbf{K}_{3}^{d}$, one needs to add the rule E4:

Lemma 16. ${ }^{10}$

$$
\forall \bar{x} \exists ! \grave{y} A \vdash_{\bar{\nabla} K_{3}^{d}[E 4]} \forall \bar{x} \exists ! \overline{\bar{y}} A .
$$

${ }^{9}$ I leave it to the reader to verify these claims.

${ }^{10}$ The following proof uses both MR1 and MR2. Written out as a nested list in full detail it can be written on the following form:

$$
\left\langle{ }_{0} 1,2,\left\langle_{1} 3,4,5,\left\langle\left\langle{ }_{2} 3,4,6,7,8_{2}\right\rangle,\left\langle_{3} 4,9,10,11_{3}\right\rangle\right\rangle, 12,13,14,15_{1}\right\rangle, 16,17_{0}\right\rangle
$$

The lists $\left\langle_{2} \ldots{ }_{2}\right\rangle$ and $\left\langle_{3} \ldots 3\right\rangle$ are the MR1-subproofs, whereas the nested list $\left\langle_{1} \ldots\right.$ is the MR2-subproof. 
Proof.

$\begin{array}{lcl}\text { (1) } & \forall \bar{x} \exists y(A(\bar{x}, y) \wedge \forall z(A(\bar{x}, z) \supset z \doteq y)) & \text { assumption } \\ \text { (2) } \quad \exists y(A(\bar{x}, y) \wedge \forall z(A(\bar{x}, z) \supset z \doteq y)) & 1, \mathrm{Q} 1 \\ \text { (3) } & A(\bar{x}, c) \wedge \forall z(A(\bar{x}, z) \supset z \doteq c) & \text { assumption for MR2 } \\ \text { (4) } & A(\bar{x}, c) & 3, \mathrm{R} 2 \\ \text { (5) } & \neg A(\bar{x}, z) \vee z \doteq c & 3, \mathrm{Q} 1, \mathrm{R} 2, \text { \& def. of } \supset \\ \text { (6) } & \neg A(\bar{x}, z) & \text { assumption for MR1 } \\ \text { (7) } & z \neq c & 4,6, \mathrm{E} 4 \\ \text { (8) } & z \doteq c \supset A(\bar{x}, z) & 7, \mathrm{R} 3, \text { def. of } \supset \\ \text { (9) } & z \doteq c & \text { assumption for MR1 } \\ \text { (10) } & A(\bar{x}, z) & 4,9, \mathrm{E} 2 \\ \text { (11) } & z \doteq c \supset A(\bar{x}, z) & 10, \mathrm{R} 3, \text { def. of } \supset \\ \text { (12) } \quad z \doteq c \supset A(\bar{x}, z) & 5-11, \mathrm{MR} 1 \\ \text { (13) } \quad A(\bar{x}, z) \equiv z \doteq c & 5,12, \mathrm{R} 1 \\ \text { (14) } \quad \forall z(A(\bar{x}, z) \equiv z \doteq c) & 3-13, \mathrm{RQ} \\ \text { (15) } \quad \exists y \forall z(A(\bar{x}, z) \equiv z \doteq y) & 14, \mathrm{Q} 3 \\ \text { (16) } \exists y \forall z(A(\bar{x}, z) \equiv z \doteq y) & 2-15, \mathrm{MR} 2 \\ \text { (17) } \forall \bar{x} \exists y \forall z(A(\bar{x}, z) \equiv z \doteq y) & 16, \mathrm{RQ}\end{array}$

There are two factors which make the two definitions of $\exists$ ! less than optimal; first that $\forall \bar{x} \exists ! y A+\forall \bar{x} \forall y(\neg A \vee A)$ holds in $\bar{\forall} \mathbf{K}_{3}^{d}$. Thus one can't state that there is one and only one $A$, unless excluded middle holds for $A$. Secondly, the conditional, $\supset$, used in both definitions of $\exists$ ! does not obey modus ponens in $\bar{\nabla} \mathbf{L} \mathbf{P}^{d}$. Note furthermore that $\bar{\nabla} \mathbf{L} \mathbf{P}^{d}$ not only lacks modus ponens for $\supset$, but has no definable implication-like connective $\rightarrow$ for which modus ponens holds ([4, Thm. 4.1]). This, as we will see, severely restricts how one can reason with Skolem functions in $\bar{\nabla} \mathbf{L} \mathbf{P}^{d}$.

Most of the results from here on out will be on functional formulas, and it will be useful to in fact add such a rule to the logic in question, and not just consider if it can be added conservatively.

Definition 21. If $\mathbf{L}$ is a logic, then $\mathbf{L}\left[\mathcal{S}^{\supset}\right]$ is $\mathbf{L}$ augmented with the following restricted version of the Skolem rule:

$$
\left(\mathcal{S}^{\supset}\right) \forall \bar{x} \exists ! \bar{y} A(\bar{x}, y) \vdash \forall \bar{x}\left(A\left(\bar{x}, f_{A}(\bar{x})\right) \wedge \forall y\left(A(\bar{x}, y) \supset y \doteq f_{A}(\bar{x})\right)\right)
$$

Obviously, $\mathcal{S}^{\supset}$ is simply a restricted form of $\mathcal{S}$, and so can also be added conservatively. Furthermore, since

$$
\begin{aligned}
& \Theta^{s f_{\omega}^{\supset}} \vdash_{\bar{\nabla} \mathbf{K}_{3}^{d}} A \Longleftrightarrow \Theta \vdash_{\bar{\nabla} \mathbf{L} \mathbf{P}^{d}\left[\mathcal{S}^{\supset}\right]} A \\
& \Theta^{s f_{\omega}^{\supset}} \vdash_{\bar{\nabla} \mathbf{K}_{3}^{d}[E 3]} A \Longleftrightarrow \Theta \vdash_{\bar{\nabla} \mathbf{K}_{3}^{d}\left[\mathcal{S}^{\supset}, E 3\right]} A
\end{aligned}
$$

Australasian Journal of Logic (14:1) 2017, Article no. 7 
where $\Theta^{s f_{\omega}^{7}}$ is got by modifying the construction in Thm. 9 so as to only introduce Skolem functions for functional formulas, and any $\bar{\nabla} \mathbf{K}_{3}^{d}-/ \overline{\bar{\nabla}} \mathbf{K}_{3}^{d}[E 3]-$ model for $\Theta^{s f_{\omega}^{\supset}}$ can obviously be generated using that construction, we have the following corollary to Fact 1 :

Fact 2. If $\mathfrak{M}$ is a class of models, then $\mathfrak{M}^{s f_{\omega}}$ is the class of models generated from $\mathfrak{M}$ using Thm. 9.

(1) $\bar{\nabla} \mathbf{K}_{3}^{d}[\mathcal{S}]$ and $\bar{\nabla} \mathbf{K}_{3}^{d}\left[\mathcal{S}^{\supset}\right]$ are both sound and complete with regards to $\mathfrak{M}^{s f_{\omega}}$ where $\mathfrak{M}$ is the class of $\bar{\nabla} \mathbf{K}_{3}^{d}$-models

(2) $\bar{\nabla} \mathbf{K}_{3}^{d}[\mathcal{S}, E 3]$ and $\bar{\nabla} \mathbf{K}_{3}^{d}\left[\mathcal{S}^{\supset}, E 3\right]$ are both sound and complete with regards to $\mathfrak{M}^{s f_{\omega}}$ where $\mathfrak{M}$ is the class of $\bar{\nabla} \mathbf{K}_{3}^{d}[E 3]$-models.

\section{Definable Skolem functions in Strong Kleene Logic}

This section shows how definable Skolem functions behave in $\bar{\nabla} \mathbf{K}_{3}^{d}$ and $\bar{\nabla} \mathbf{K}_{3}^{d}[E 3]$. It is shown that $\bar{\nabla} \mathbf{K}_{3}^{d}$ is semifit for Skolem functions relative to $\exists !^{2}$, fit relative to $\exists !^{\prime}$, and that $\bar{\nabla} \mathbf{K}_{3}^{d}[E 3]$ is fit relative to both. It is also shown that there is a translation from $\mathcal{L}_{S_{\omega}}$ to $\mathcal{L}$ which preserves derivability.

Theorem 17. The rules

$$
\begin{aligned}
& \text { Skōlemizer } \quad \forall \bar{x} \exists ! \overline{\bar{y}} A(\bar{x}, y), \psi_{A(\bar{t}, s)} \vdash \psi_{s=f_{A}(\bar{t})} \\
& \text { deSkōlemizer } \quad \forall \bar{x} \exists ! \overline{\bar{y}} A(\bar{x}, y), \psi_{s=f_{A}(\bar{t})} \vdash \psi_{A(\bar{t}, s)}
\end{aligned}
$$

are derivable in $\bar{\nabla} \mathbf{K}_{3}^{d}\left[\mathcal{S}^{\supset}\right]$.

Proof. Let $\mathfrak{A}_{\mathfrak{s}}$ be an arbitrary model for $\bar{\nabla} \mathbf{K}_{3}^{d}\left[\mathcal{S}^{\supset}\right]$, and assume that $\mathfrak{A}_{\mathfrak{s}} \mathrm{F}$ $\forall \bar{x} \exists ! \overline{\bar{y}} A(\bar{x}, y)$. The truth-table for $\equiv$ is

$$
\begin{array}{c|ccc}
\equiv & \perp & \mathbf{n} & \top \\
\hline \perp & \top & \mathbf{n} & \perp \\
\mathbf{n} & \mathbf{n} & \mathbf{n} & \mathbf{n} \\
\top & \perp & \mathbf{n} & \top,
\end{array}
$$

and so $\mathfrak{A}_{\mathfrak{S}}$ has to assign the same classical value to $A(\bar{t}, s)$ and $s \doteq f_{A}(\bar{t})$. Since $\mathfrak{H}_{\mathfrak{s}}$ is compositional it follows that $\mathfrak{H}_{\mathfrak{s}}\left(\psi_{A(\bar{t}, s)}\right)=\mathfrak{H}_{\mathfrak{s}}\left(\psi_{s=f_{A}(\bar{t})}\right)$ which suffices for showing that $\mathfrak{A}_{\mathfrak{s}}$ validates both deSkōlemizer and Skōlemizer. Since $\mathfrak{A}_{\mathfrak{s}}$ was arbitrary it follows that any $\bar{\nabla} \mathbf{K}_{3}^{d}\left[\mathcal{S}^{\supset}\right]$-model validates these rules, and so by the completeness theorem for $\bar{\nabla} \mathbf{K}_{3}^{d}\left[\mathcal{S}^{\supset}\right]$ it follows that they are derivable in $\bar{\nabla} \mathbf{K}_{3}^{d}\left[\mathcal{S}^{\supset}\right]$.

Corollary 18. $\bar{\nabla} \mathbf{K}_{3}^{d}$ is fit for Skolem functions relative to $\overline{\bar{\Xi}}$ !.

Corollary 19. $\bar{\nabla} \mathbf{K}_{3}^{d}[E 3]$ is fit for Skolem functions relative to both $\overline{\bar{\exists} !}$ and قै!. 
Does this solve the problem for $\bar{\nabla} \mathbf{K}_{3}^{d}$ ? Let's assume that one is able to derive $\forall \bar{x} \exists ! y A(\bar{x}, y)$. In order to make use of either deSkôlemizer or Skôlemizer, one would first need to derive $\forall \bar{x} \exists ! ! \bar{y} A(\bar{x}, y)$, but in order to do so one generally needs E4 to hold as Lem. 16 above shows. Without E4 it seems needlessly difficult to prove unique existential statements; the theory would need to entail the relevant instances of E4 by itself. One could of course simply add E4 or E3 as an extra rule. However, E4, which intuitively says that distinguishable objects are non-identical, is sometimes rejected on account that it rules out indeterminate identity. ${ }^{11}$ Regardless of this it seems worth while to investigate the consequences of using $\exists$ ! as the definition of $\exists$ ! before scrapping $\bar{\nabla} \mathbf{K}_{3}^{d}$ for the stronger logic $\bar{\nabla} \mathbf{K}_{3}^{d}[E 3]$. The next results show that $\bar{\nabla} \mathbf{K}_{3}^{d}$ is semifit for Skolem functions, but not fit simpliciter.

Theorem 20. The rule

$$
\text { deSkỏlemizer } \forall \bar{x} \exists ! y A(\bar{x}, y), \psi_{s=f_{A}(\bar{t})} \vdash \psi_{A(\bar{t}, s)}
$$

is derivable in $\bar{\nabla} \mathbf{K}_{3}^{d}\left[\mathcal{S}^{\supset}\right]$.

Proof. Assume that $\mathfrak{U}_{\mathfrak{s}}$ is an arbitrary model for $\bar{\nabla} \mathbf{K}_{3}^{d}$ and extend it to a model for $\mathcal{S}^{\supset}$. Assume that $\mathfrak{H}_{\mathfrak{s}}^{s_{f_{\omega}}} \vDash \forall \bar{x} \exists ! y \bar{y} A(\bar{x}, y)$. Since the model is arbitrary it will follow from Fact 2 that the rule is in fact derivable if we can show that

$$
\mathfrak{H}_{\mathfrak{s}}^{s f_{\omega}}\left(\psi_{s=f_{A}(\bar{t})}\right)=\mathrm{T} \Rightarrow \mathfrak{H}_{\mathfrak{s}}^{s f_{\omega}}\left(\psi_{A(\bar{t}, s)}\right)=\mathrm{T} .
$$

In light of Thm. 3 above it will suffice to show that it is always the case that

$$
\mathfrak{H}_{\mathfrak{s}}^{s f_{\omega}}\left(s \doteq f_{A}(\bar{t})\right) \leq \mathfrak{H}_{\mathfrak{s}}^{s f_{\omega}}(A(\bar{t}, s)) .
$$

I will therefore show the following:

$$
\begin{aligned}
& \text { (1) } \mathfrak{H}_{\mathfrak{s}}^{s_{f_{\omega}}}\left(s \doteq f_{A}(\bar{t})\right)=\mathrm{T} \Rightarrow \mathfrak{H}_{\mathfrak{s}}^{s f_{\omega}}(A(\bar{t}, s))=\mathrm{T} \\
& \text { (2) } \mathfrak{H}_{\mathfrak{s}}^{f_{\mathfrak{s}}}\left(s \doteq f_{A}(\bar{t})\right)=\perp \Rightarrow \mathfrak{H}_{\mathfrak{s}}^{f_{\omega_{\omega}}}(A(\bar{t}, s))=\perp \\
& \text { (3) } \mathfrak{H}_{\mathfrak{s}}^{f_{\omega}}\left(s \doteq f_{A}(\bar{t})\right)=\mathbf{n} \Rightarrow \mathfrak{U}_{\mathfrak{s}}^{f_{\mathfrak{s}}}(A(\bar{t}, s))=\perp
\end{aligned}
$$

Assume that $\mathfrak{H}_{s}^{s f_{\omega}}\left(s \doteq f_{A}(\bar{t})\right)=\mathrm{T}$. The Skolem rule together with the fact that $\mathfrak{I}_{\mathfrak{s}}^{s f_{\omega}}(\forall \bar{x} \exists ! y A(\bar{x}, y))=T$ entails that $\mathfrak{Q}_{\mathfrak{s}}^{s f_{\omega}}\left(A\left(\bar{t}, f_{A}(\bar{t})\right)\right)=T$. Since $\mathfrak{H}_{\mathfrak{s}}^{s f_{\omega}}$ validates E2 it follows that $\mathfrak{H}_{\mathfrak{s}}^{s f_{\omega}}(A(\bar{t}, s))=\mathrm{T}$. Assume that $\mathfrak{H}_{\mathfrak{s}}^{s f_{\omega}}\left(s \doteq f_{A}(\bar{t})\right)=\perp$. The Skolem rule together with the fact that $\mathfrak{H}_{\mathfrak{s}}^{s f_{\omega}}(\forall \bar{x} \exists ! y \bar{y} A(\bar{x}, y))=T$ entails that $\mathfrak{H}_{\mathfrak{s}}^{s f_{\omega}}\left(A(\bar{t}, s) \supset s \doteq f_{A}(\bar{t})\right)=\mathrm{T}$, and so $\mathfrak{H}_{\mathfrak{s}}^{s f_{\omega}}(A(\bar{t}, s))=\perp$. Lastly, assume that $\mathfrak{U}_{\mathfrak{s}}^{s_{\omega}}\left(s \doteq f_{A}(\bar{t})\right)=\mathbf{n}$. Since $\mathfrak{U}_{\mathfrak{s}}^{s_{\omega}}\left(A(\bar{t}, s) \supset s \doteq f_{A}(\bar{t})\right)=\mathrm{T}$, it follows that $\mathfrak{A}_{\mathfrak{s}}^{s f_{\omega}}(A(\bar{t}, s))=\perp$.

Corollary 21. $\bar{\nabla} \mathbf{K}_{3}^{d}$ is semifit for Skolem functions relative to $\exists$ ヨ!.

\footnotetext{
${ }^{11}$ See [15, ch. 3] for a discussion.
}

Australasian Journal of Logic (14:1) 2017, Article no. 7 
Theorem 22. $\bar{\nabla} \mathbf{K}_{3}^{d}$ is semifit only for Skolem functions relative to $\exists$ ق!.

Proof. I will first show that $\exists x \forall y(y \neq x \vee y \doteq x)$ is derivable from $\forall x \exists ! y A(x, y)$ using the Skollemizer and then show that this amounts to a non-conservative extension.

(1) $\forall x \exists ! y A(x, y)$

assumption

(2) $\forall x\left(A\left(x, f_{A}(x)\right) \wedge \forall y\left(A(x, y) \supset y \doteq f_{A}(x)\right)\right)$ 1, Skolem rule

(3) $\forall x \forall y\left(A(x, y) \supset y \doteq f_{A}(x)\right)$

2, fiddling

(4) $\forall x \forall y\left(y \doteq f_{A}(x) \supset y \doteq f_{A}(x)\right)$

3, Skolemizer

(5) $\exists x \forall y(y \not \dot{\neq} x \vee y \doteq x)$

4, def. of $\supset$, Q1 \& Q3

The countermodel to $\exists x \forall y(y \dot{\neq} x \vee y \doteq x)$ is as follows: let $|\mathfrak{A}|$ be the set $\{a, b\}$, and let $\doteq$ and the non-logical predicate $A(x, y)$ be interpreted according to the following matrices:

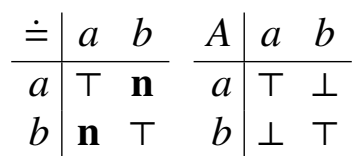

It is easy to verify that $\mathfrak{A}_{\mathfrak{5}}(\forall x \exists ! y A(x, y))=\top$, but that $\mathfrak{A}_{\mathfrak{5}}(\exists x \forall y(y \dot{=} x \vee y \dot{\neq} x))=$ n. By the soundness theorem for $\bar{\nabla} \mathbf{K}_{3}^{d}$ it follows that adding Skỏlemizer to $\bar{\nabla} \mathbf{K}_{3}^{d}$ yields a non-conservative extension.

The next goal is to show that there is a translation from the Skolemextended language $\mathcal{L}_{s_{\omega}}$ to $\mathcal{L}$ which preserves derivability in both $\bar{\nabla} \mathbf{K}_{3}^{d}\left[\mathcal{S}^{\supset}\right]$ and $\bar{\nabla} \mathbf{K}_{3}^{d}\left[\mathcal{S}^{\supset}, E 3\right]$. The theorem relies on the following lemma:

Lemma 23. If $f_{A}$ has been introduced by $\mathcal{S}^{\supset}$, then for any formula $B(y)$ where $y$ does not occur in $A\left(f_{A}(\bar{t})\right)$ and any $\bar{\nabla} \mathbf{K}_{3}^{d}$-model $\mathfrak{A}_{\mathfrak{s}}$,

$$
\mathfrak{A}_{\mathfrak{s}}^{s f_{\omega}}(\exists y(A(\bar{t}, y) \wedge B(y)))=\mathfrak{A}_{\mathfrak{s}}^{s f_{\omega}}\left(B\left(f_{A}(\bar{t})\right)\right)
$$

where $\mathfrak{H}_{\mathfrak{s}}^{s f_{\omega}}$ is any model got from $\mathfrak{A}_{\mathfrak{s}}$ using the construction in Thm. 9.

Proof. The proof is divided into six subproofs:

(1) $\mathfrak{A}_{\mathfrak{s}}^{s f_{\omega}}(\exists y(A(\bar{t}, y) \wedge B(y)))=\top \Rightarrow \mathfrak{A}_{\mathfrak{s}}^{s f_{\omega}}\left(B\left(f_{A}(\bar{t})\right)\right)=\top$

(2) $\quad \mathfrak{U}_{\mathfrak{s}}^{s f_{\omega}}\left(B\left(f_{A}(\bar{t})\right)\right)=\top \Rightarrow \mathfrak{A}_{\mathfrak{s}}^{s f_{\omega}}(\exists y(A(\bar{t}, y) \wedge B(y)))=\top$

(3) $\mathfrak{A}_{\mathfrak{s}}^{s f_{\omega}}(\exists y(A(\bar{t}, y) \wedge B(y)))=\perp \Rightarrow \mathfrak{A}_{\mathfrak{s}}^{s f_{\omega}}\left(B\left(f_{A}(\bar{t})\right)\right)=\perp$

(4) $\quad \mathfrak{A}_{\mathfrak{s}}^{s f_{\omega}}\left(B\left(f_{A}(\bar{t})\right)\right)=\perp \Rightarrow \mathfrak{A}_{\mathfrak{s}}^{s f_{\omega}}(\exists y(A(\bar{t}, y) \wedge B(y)))=\perp$

(5) $\mathfrak{A}_{\mathfrak{s}}^{s f_{\omega}}(\exists y(A(\bar{t}, y) \wedge B(y)))=\mathbf{n} \Rightarrow \mathfrak{A}_{\mathfrak{s}}^{s f_{\omega}}\left(B\left(f_{A}(\bar{t})\right)\right)=\mathbf{n}$

(6) $\quad \mathfrak{A}_{\mathfrak{s}}^{s f_{\omega}}\left(B\left(f_{A}(\bar{t})\right)\right)=\mathbf{n} \Rightarrow \mathfrak{A}_{\mathfrak{s}}^{s f_{\omega}}(\exists y(A(\bar{t}, y) \wedge B(y)))=\mathbf{n}$

It is plain to see that (2), (4) and (6) follow from (1), (3) and (5) the proof of which will make frequent use of the following:

Australasian Journal of Logic (14:1) 2017, Article no. 7 


$$
\begin{aligned}
& \text { (I) } \mathfrak{U}_{\mathfrak{s}}^{s_{f_{\omega}}}\left(A\left(\bar{t}, f_{A}(\bar{t})\right)\right)=\top \\
& \text { (II) } \mathfrak{U}_{\mathfrak{s}}^{f_{f_{\omega}}}\left(\forall y\left(A(\bar{t}, y) \supset y \doteq f_{A}(\bar{t})\right)\right)=\mathrm{T}
\end{aligned}
$$

(1). Goal: $\mathfrak{N}_{\mathfrak{s}}^{s f_{\omega}}(\exists y(A(\bar{t}, y) \wedge B(y)))=\mathrm{T} \Rightarrow \mathfrak{U}_{\mathrm{s}}^{s f_{\omega}}\left(B\left(f_{A}(\bar{t})\right)\right)=\mathrm{T}$
(1) $\mathfrak{H}_{\mathfrak{s}}^{s f_{\omega}}(\exists y(A(\bar{t}, y) \wedge B(y)))=\top$ assumption
(2) $\mathfrak{H}_{s(y / a)}^{s f_{\omega}}(A(\bar{t}, y) \wedge B(y))=T \quad$ for some $a \in|\mathfrak{A}|$
(3) $\mathfrak{H}_{\mathfrak{s}(y / a)}^{s f_{\omega}}\left(A(\bar{t}, y) \supset y \doteq f_{A}(\bar{t})\right)=\top$ (II)
(4) $\mathfrak{H}_{s^{\prime}(y / a)}^{s_{\omega}}(A(\bar{t}, y))=\mathrm{T} \quad 2$
(5) $\mathfrak{H}_{\mathrm{s}(\mathrm{y} / a)}^{\mathrm{s} f_{\omega}}\left(y \doteq f_{A}(\bar{t})\right)=\mathrm{T} \quad 3,4, \mathrm{R} 7$
(6) $\mathfrak{H}_{\mathfrak{s}(y / a)}^{S_{\omega}(B(y))=T} \quad 2$
(7) $\mathfrak{H}_{\mathfrak{s}(y / /)(}^{S_{\omega} f_{\omega}}\left(B\left(f_{A}(\bar{t})\right)\right)=\mathrm{T} \quad 5,6, \mathrm{E} 2$
(8) $\mathfrak{I I}_{\mathfrak{s}}^{s f_{\omega}}\left(B\left(f_{A}(\bar{t})\right)\right)=\top \quad 7$

(3). Goal: $\mathfrak{U}_{\mathfrak{s}}^{s f_{\omega}}(\exists y(A(\bar{t}, y) \wedge B(y)))=\perp \Rightarrow \mathfrak{V}_{\mathfrak{s}}^{s f_{\omega}}\left(B\left(f_{A}(\bar{t})\right)\right)=\perp$
(1) $\mathfrak{H}_{\mathfrak{5}}^{s f_{\omega}}(\exists y(A(\bar{t}, y) \wedge B(y)))=\perp \quad$ assumption
(2) $\mathfrak{H}_{\mathfrak{5}}^{s f_{\omega}}\left(A\left(\bar{t}, f_{A}(\bar{t})\right) \wedge B\left(f_{A}(\bar{t})\right)\right)=\perp 1$
(3) $\mathfrak{I}_{\mathfrak{s}}^{s f_{\omega}}\left(A\left(\bar{t}, f_{A}(\bar{t})\right)\right)=T$
(4) $\mathfrak{H}_{\mathfrak{s}}^{s f_{\omega}}\left(B\left(f_{A}(\bar{t})\right)\right)=\perp$
2,3

(5). Goal: $\mathfrak{T}_{\mathfrak{s}}^{s f_{\omega}}(\exists y(A(\bar{t}, y) \wedge B(y)))=\mathbf{n} \Rightarrow \mathfrak{U}_{\mathfrak{s}}^{s f_{\omega}}\left(B\left(f_{A}(\bar{t})\right)\right)=\mathbf{n}$
(1) $\mathfrak{A}_{\mathfrak{s}}^{s f_{\omega}}(\exists y(A(\bar{t}, y) \wedge B(y)))=\mathbf{n}$
assumption
(2) $\mathfrak{A}_{\mathfrak{s}}^{s f_{\omega}}\left(A\left(\bar{t}, f_{A}(\bar{t})\right)\right)=\top$
(I)
(3) $\mathfrak{A}_{\mathfrak{s}}^{s f_{\omega}}\left(B\left(f_{A}(\bar{t})\right)\right) \in\{\mathbf{n}, \perp\}$
1,2
(4) $\mathfrak{Y}_{\mathfrak{s}}^{s f_{\omega}}\left(B\left(f_{A}(\bar{t})\right)\right)=\perp$ assumption for MR1
(5) $\quad \mathfrak{A}_{\mathfrak{s}(y / a)}^{s f_{\omega}}\left(\neg A(\bar{t}, y) \vee y \doteq f_{A}(\bar{t})\right)=\top$ (II), def. of $\supset$, for any $a \in|\mathfrak{A}|$$$
\mathfrak{U}_{\mathfrak{s}(y / a)}^{s f_{\omega}}\left(y \doteq f_{A}(\bar{t})\right)=\top
$$ assumption for MR1$$
\mathfrak{A}_{\mathfrak{s}(y / a)}^{s f_{\omega}}(B(y))=\perp
$$$$
\text { 4, 6, E2 }
$$$$
\mathfrak{U}_{\mathfrak{s}(y / a)}^{s f_{\omega}}(A(\bar{t}, y) \wedge B(y))=\perp \quad 8
$$$$
\mathfrak{H}_{\mathfrak{s}(y / a)}^{s f_{\omega}}(A(\bar{t}, y))=\perp
$$ 8 assumption for MR1 $\mathfrak{U}_{\mathfrak{s}(y / a)}^{s f_{\omega}}(A(\bar{t}, y) \wedge B(y))=\perp$ 9 $\mathfrak{U}_{\mathfrak{s}(y / a)}^{s f_{\omega}}(A(\bar{t}, y) \wedge B(y))=\perp$ 5, 6-10, MR1, for any $a \in|\mathfrak{A}|$ $\mathfrak{A}_{\mathfrak{s}}^{s f_{\omega}}(\exists y(A(\bar{t}, y) \wedge B(y)))=\perp$ contradiction 11 1,12
(14) $\mathfrak{A}_{\mathfrak{s}}^{s f_{\omega}}\left(B\left(f_{A}(\bar{t})\right)\right)=\mathbf{n}$ 3, 4-13, MR1

Australasian Journal of Logic (14:1) 2017, Article no. 7 
Theorem 24. There is a translation $*: \mathcal{L}_{s_{\omega}} \mapsto \mathcal{L}$ such that for any set $\Theta \subseteq \mathcal{L}$ and formula $A \in \mathcal{L}_{s_{\omega}}$,

$$
\begin{aligned}
\Theta \vdash_{\bar{\nabla}} \mathbf{K}_{3}^{d}\left[\mathcal{S}^{\supset}\right] & \Longleftrightarrow \Theta \vdash_{\bar{\nabla}} A \mathbf{K}_{3}^{d} A^{*} \\
\Theta \vdash_{\bar{\nabla} \mathbf{K}_{3}^{d}\left[\mathcal{S}^{\supset}, E 3\right]} A & \Longleftrightarrow \Theta \vdash_{\bar{\nabla} \mathbf{K}_{3}^{d}[E 3]} A^{*}
\end{aligned}
$$

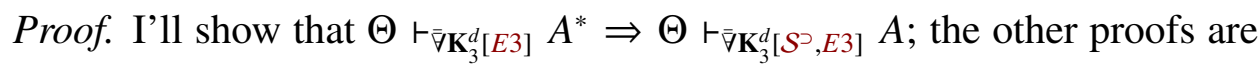
similar.

Inductively replace every atomic subformula $C\left(y / f_{B}(\bar{t})\right)$ of $A$, where $C(y)$ and $\bar{t}$ do not contain any Skolem functions, with the formula $\exists y(B(\bar{t}, y) \wedge$ $C(y)$ ), and let the resulting formula be $A^{*}$. Assume that $\Theta \vdash_{\bar{\nabla}} \mathbf{K}_{3}^{d}[E 3]$ $\mathfrak{A}_{\mathfrak{5}}$ be an arbitrary $\bar{\nabla} \mathbf{K}_{3}^{d}[E 3]$-model such that $\mathfrak{A}_{\mathfrak{5}}(\theta)=\mathrm{T}$ for every $\theta \in \Theta$. By the soundness theorem for $\bar{\nabla} \mathbf{K}_{3}^{d}[E 3]$ it follows that $\mathfrak{A}_{\mathfrak{5}}\left(A^{*}\right)=\mathrm{T}$. Extend $\mathfrak{A}_{\mathfrak{5}}$ to $\mathfrak{H}_{\mathfrak{s}}^{s f_{\omega}}$ using Thm. 9. From Lem. 13 it follows that $\mathfrak{H}_{\mathfrak{s}}^{s f_{\omega}}$ is a model for $\bar{\nabla} \mathbf{K}_{3}^{d}\left[\mathcal{S}^{\supset}, E 3\right]$. Furthermore, $\mathfrak{H}_{\mathfrak{s}}^{s f_{\omega}}(\theta)=\mathrm{T}$ for every $\theta \in \Theta$, and $\mathfrak{A}_{\mathfrak{s}}^{s f_{\omega}}\left(A^{*}\right)=\mathrm{T}$. From Lem. 23 it follows that $\mathfrak{A}_{\mathfrak{s}}^{s f_{\omega}}(A)=T$. Since $\mathfrak{A}_{\mathfrak{s}}$ was arbitrary, it follows

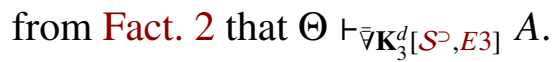

I have shown in this section that $\bar{\nabla} \mathbf{K}_{3}^{d}$ does quite well when it comes to Skolem functions: it both suffices for the existence of a translation which preserves derivability both to and from the definable Skolem function extended language, and one can always substitute $A(\bar{t}, s)$ for the Skolem equation $s \doteq f_{A}(\bar{t})$, although not the other way around; $; \bar{\nabla} \mathbf{K}_{3}^{d}$ is only semifit for Skolem functions. $\bar{\nabla} \mathbf{K}_{3}^{d}[E 3]$ was shown to be not only semifit for Skolem functions, but fit simpliciter. The downside to using $\bar{\nabla} \mathbf{K}_{3}^{d}[E 3]$, however, is that all identity statements are classical in $\bar{\nabla} \mathbf{K}_{3}^{d}[E 3]$.

\section{Definable Skolem functions in The Logic of Paradox}

I will in this section show that the translation used in the above section, also works for $\bar{\nabla} \mathbf{L} \mathbf{P}^{d}$ although not quite as well, and that $\bar{\nabla} \mathbf{L} \mathbf{P}^{d}$ is unfit for Skolem functions relative to both definitions of $\exists$ !. 
Lemma 25. If $f_{A}$ has been introduced by $\mathcal{S}^{\supset}$, then for any formula $B$ in which $f_{A}(\bar{t})$ is substitutable for $y$ and any $\bar{\nabla} \mathbf{L} \mathbf{P}^{d}$-model $\mathfrak{A}_{\mathfrak{s}}$,

(2) $\mathfrak{U}_{\mathrm{s}}^{s f_{\omega}}(\exists y(A(\bar{t}, y) \wedge B(y)))=\top \Rightarrow \mathfrak{U}_{\mathrm{s}}^{s f_{\omega}}\left(B\left(f_{A}(\bar{t})\right)\right)=\top$

(3) $\quad \mathfrak{H}_{\mathfrak{s}}^{s f_{\omega}}\left(B\left(f_{A}(x)\right)\right)=\perp \Rightarrow \mathfrak{H}_{\mathfrak{s}}^{s f_{\omega}}(\exists y(A(x, y) \wedge B(y))) \in\{\mathbf{b}, \perp\}$

(4) $\mathfrak{U}_{\mathfrak{s}}^{s f_{\omega}}(\exists y(A(\bar{t}, y) \wedge B(y)))=\perp \Rightarrow \mathfrak{U}_{5}^{s f_{\omega}}\left(B\left(f_{A}(\bar{t})\right)\right)=\perp$

(5) $\quad \mathfrak{H}_{\mathfrak{s}}^{s f_{\omega}}\left(B\left(f_{A}(x)\right)\right)=\mathbf{b} \Rightarrow \mathfrak{H}_{\mathfrak{s}}^{s f_{\omega}}(\exists y(A(x, y) \wedge B(y)))=\mathbf{b}$

(6) $\mathfrak{H}_{\mathfrak{s}}^{s f_{\omega}}(\exists y(A(\bar{t}, y) \wedge B(y)))=\mathbf{b} \Rightarrow \mathfrak{I}_{\mathfrak{s}}^{s f_{\omega}}\left(B\left(f_{A}(\bar{t})\right)\right) \in\{\perp, \mathbf{b}, \top\}$

where $\mathfrak{U}_{\mathfrak{5}}^{s f_{\omega}}$ is any model got from $\mathfrak{A}_{\mathfrak{5}}$ using the construction in Thm. 9.

Proof.

$$
\begin{aligned}
& \text { (I) } \mathfrak{H}_{\mathfrak{s}}^{s_{\omega}}\left(A\left(\bar{t}, f_{A}(\bar{t})\right)\right) \in\{\mathbf{b}, \top\} \\
& \text { (II) } \mathfrak{H}_{\mathfrak{s}}^{f_{\omega}}\left(\forall y\left(A(\bar{t}, y) \supset y \doteq f_{A}(\bar{t})\right)\right) \in\{\mathbf{b}, \top\}
\end{aligned}
$$

(1). Goal: $\mathfrak{H}_{\varsigma}^{s f_{\omega}}\left(B\left(f_{A}(x)\right)\right)=\top \Rightarrow \mathfrak{U}_{\varsigma}^{s f_{\omega}}(\exists y(A(x, y) \wedge B(y))) \in\{\mathbf{b}, \top\}$
(1) $\mathfrak{U}_{\mathrm{s}}^{s_{\omega}}\left(B\left(f_{A}(x)\right)\right)=\mathrm{T}$ assumption
(2) $\mathfrak{U}_{5}^{s_{\omega}}\left(A\left(x, f_{A}(x)\right)\right) \in\{\mathbf{b}, \top\}$
(3) $\mathfrak{H}_{\mathfrak{s}}^{s f_{\omega}}\left(A\left(x, f_{A}(x)\right) \wedge B\left(f_{A}(x)\right)\right) \in\{\mathbf{b}, \top\} \quad 1,2$
(4) $\mathfrak{U}_{\mathfrak{s}}^{s f_{\omega}}(\exists y(A(x, y) \wedge B(y))) \in\{\mathbf{b}, T\}$
3

(2). Goal: $\mathfrak{H}_{\mathfrak{s}}^{s f_{\omega}}(\exists y(A(\bar{t}, y) \wedge B(y)))=\top \Rightarrow \mathfrak{U}_{\mathfrak{s}}^{s f_{\omega}}\left(B\left(f_{A}(\bar{t})\right)\right)=\top$
(1) $\mathfrak{U}_{s}^{s f_{\omega}}(\exists y(A(\bar{t}, y) \wedge B(y)))=\mathrm{T}$ assumption
(2) $\mathfrak{U}_{\mathfrak{s}(y / a)}^{s f_{\omega}}(A(\bar{t}, y) \wedge B(y))=\top$ for some $a \in|\mathfrak{A}|$

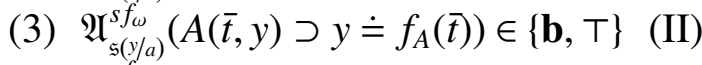
(4) $\mathfrak{H}_{\mathfrak{s}(y / a)}^{s f_{\omega}}(A(\bar{t}, y))=\top$ 2
(5) $\mathfrak{H}_{\mathfrak{s}(y / /)}^{s_{\omega} f_{\omega}}\left(y \doteq f_{A}(\bar{t})\right) \in\{\mathbf{b}, T\}$ 3,4
(6) $\mathfrak{H}_{\mathfrak{s}(y / a)}^{s_{\omega} f_{a}}(B(y))=T$ 2
(7) $\mathfrak{H}_{\mathfrak{s}(\mathrm{y} / a)}^{S f_{(j)}}\left(B\left(f_{A}(\bar{t})\right)\right)=\mathrm{T}$ $5,6, \mathrm{E} 2$
(8) $\mathfrak{H}_{\mathfrak{s}}^{S_{\mathfrak{s}}}\left(B\left(f_{A}(\bar{t})\right)\right)=\top$ 7

(3). Goal: $\mathfrak{H}_{\mathfrak{s}}^{s f_{\omega}}\left(B\left(f_{A}(x)\right)\right)=\perp \Rightarrow \mathfrak{A}_{\mathfrak{s}}^{s f_{\omega}}(\exists y(A(x, y) \wedge B(y))) \in\{\mathbf{b}, \perp\}$.

This follows from (2).

Australasian Journal of Logic (14:1) 2017, Article no. 7 
(4). Goal: $\mathfrak{U}_{\mathfrak{s}}^{s f_{\omega}}(\exists y(A(\bar{t}, y) \wedge B(y)))=\perp \Rightarrow \mathfrak{U}_{\mathfrak{s}}^{s f_{\omega}}\left(B\left(f_{A}(\bar{t})\right)\right)=\perp$

$$
\begin{array}{lll}
\text { (1) } & \mathfrak{I}_{\mathfrak{s}}^{s_{\omega}}(\exists y(A(\bar{t}, y) \wedge B(y)))=\perp & \text { assumption } \\
\text { (2) } & \mathfrak{I}_{\mathfrak{s}}^{s_{\omega} f_{\omega}}\left(A\left(\bar{t}, f_{A}(\bar{t})\right) \wedge B\left(f_{A}(\bar{t})\right)\right)=\perp & 1 \\
\text { (3) } & \mathfrak{I}_{\mathfrak{s}}^{s_{\omega_{\omega}}}\left(A\left(\bar{t}, f_{A}(\bar{t})\right)\right) \in\{\mathbf{b}, T\} & \text { (I) } \\
\text { (4) } & \mathfrak{I}_{\mathfrak{s}}^{s f_{\omega}}\left(B\left(f_{A}(\bar{t})\right)\right)=\perp & 2,3
\end{array}
$$

(5). Goal: $\mathfrak{U}_{\mathfrak{s}}^{s_{\omega}}\left(B\left(f_{A}(t)\right)\right)=\mathbf{b} \Rightarrow \mathfrak{U}_{\mathfrak{s}}^{s_{f_{\omega}}}(\exists y(A(t, y) \wedge B(y)))=\mathbf{b}$
(1) $\mathfrak{A}_{\mathfrak{s}}^{s f_{\omega}}\left(B\left(f_{A}(t)\right)\right)=\mathbf{b}$ assumption
(2) $\mathfrak{H}_{\mathfrak{s}}^{s f_{\omega}}\left(A\left(x, f_{A}(t)\right)\right) \in\{\mathbf{b}, \top\}$
(3) $\mathfrak{U}_{5}^{s f_{\omega}}\left(A\left(x, f_{A}(t)\right) \wedge B\left(f_{A}(x)\right)\right)=\mathbf{b}$
1,2
(4) $\quad \mathfrak{U}_{\mathfrak{s}(y / a)}^{s f_{\omega}}(A(t, y) \wedge B(y))=\mathrm{T}$ assumption; for one $a \in|\mathfrak{U}|$
(5) $\quad \mathfrak{A}_{\mathfrak{s}(y / a)}^{f_{(\omega)}}\left(A(t, y) \supset y \doteq f_{A}(x)\right) \in\{\mathbf{b}, \top\}$
(6) $\quad \mathfrak{V}_{\mathfrak{s}(y / a)}^{s_{\omega} f_{\omega}}\left(y \doteq f_{A}(t)\right) \in\{\mathbf{b}, T\}$
(7) $\quad \mathfrak{H}_{\mathfrak{s}\left(y_{/ 2}\right)}^{s f_{\omega}}(B(y))=\top$
4,5
(8) $\quad \mathfrak{V}_{\mathrm{s}(y / a)}^{s f_{\omega}}\left(B\left(f_{A}(t)\right)\right)=\mathrm{T}$
4
(9)
contradiction
$6,7, \mathrm{E} 2$
1,8
(10) $\mathfrak{H}_{\mathfrak{s}}^{s f_{\omega}}(\exists y(A(t, y) \wedge B(y)))=\mathbf{b}$
$3,4-9$

(6). Goal: $\mathfrak{H}_{\mathfrak{s}}^{s f_{\omega}}(\exists y(A(\bar{t}, y) \wedge B(y)))=\mathbf{b} \Rightarrow \mathfrak{H}_{\mathfrak{s}}^{s f_{\omega}}\left(B\left(f_{A}(\bar{t})\right)\right) \in\{\perp, \mathbf{b}, \top\}$

Trivially.

Corollary 26. If $f_{A}$ has been introduced by $\mathcal{S}^{\supset}$, then for every model $\mathfrak{H}_{\mathfrak{s}}^{s f_{\omega}}$ and every formula $\psi$ and $B$ and every term $t$,

$$
\mathfrak{H}_{\mathfrak{s}}^{s f_{\omega}}\left(\psi_{\exists y(A(t, y) \wedge B(y))}\right) \leq \mathfrak{H}_{\mathfrak{s}}^{s f_{\omega}}\left(\psi_{B\left(f_{A}(t)\right)}\right) .
$$

Proof. This follows from Lem. 25 using (2), (4) and (6).

Could one improve the above relations in Lem. 25? The answer is no:

Lemma 27. There are models $\mathfrak{H}_{\mathfrak{5}}$ and Skolem-function extensions $\mathfrak{U}_{\mathfrak{5}}^{s f_{\omega}}$ thereof for $\bar{\nabla} \mathbf{L} \mathbf{P}^{d}\left[\mathcal{S}^{\supset}\right]$ such that
(1) $\mathfrak{U}_{\mathfrak{s}} \vDash \forall x \exists ! y A(x, y)$
(2) $\mathfrak{A}_{\mathfrak{5}}(\exists y(A(x, y) \wedge B(y)))=\mathbf{b}$
(3) $\mathfrak{A}_{\mathfrak{s}}^{s f_{1}}\left(B\left(f_{A}(x)\right)\right) \in\{\perp, \top\}$

Australasian Journal of Logic (14:1) 2017, Article no. 7 
Proof. Let $\mathfrak{A}$ be a model for $\bar{\nabla} \mathbf{L} \mathbf{P}^{d}$ and let it interpret the binary predicate $A(x, y)$, the unary predicate $B(y)$ and the identity predicate $\doteq$ according to the following tables:

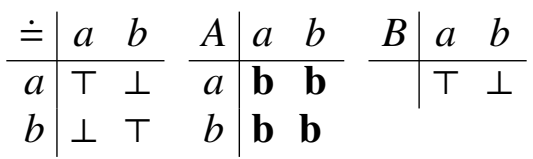

It is easy to calculate that $\mathfrak{A}_{\mathfrak{s}}(\forall x \exists ! y A(x, y))=\mathbf{b}$ for every variable assignment function $\mathfrak{s}$. Let $\mathfrak{s}$ and $\mathfrak{s}^{\prime}$ be two such functions such that $\mathfrak{s}(x)=a$, and $\mathfrak{s}^{\prime}(x)=b$. Then $\mathfrak{U}_{\mathfrak{s}}(\exists y(A(x, y) \wedge B(y)))=\mathfrak{H}_{\mathfrak{s}^{\prime}}(\exists y(A(x, y) \wedge B(y)))=\mathbf{b}$. One of the possible ways to extend $\mathfrak{A}$ into a Skolem model which validates $\bar{\nabla} \mathbf{L} \mathbf{P}^{d}\left[\mathcal{S}^{\supset}\right]$, is to interpret $f_{A}$ as the identity function. It then follows that $\mathfrak{A}_{\mathfrak{s}}^{s f_{1}}\left(B\left(f_{A}(x)\right)\right)=\top$ and $\mathfrak{U}_{\mathfrak{s}^{\prime}}^{s f_{1}}\left(B\left(f_{A}(x)\right)\right)=\perp$.

Theorem 28. There is a translation $*: \mathcal{L}_{s_{\omega}} \mapsto \mathcal{L}$ such that for any set $\Theta \subseteq \mathcal{L}$ and formula $A \in \mathcal{L}_{s_{\omega}}$,

$$
\Theta \vdash_{\bar{\nabla} \mathbf{L} \mathbf{P}^{d}\left[\mathcal{S}^{\supset}\right]} A \Longrightarrow \Theta \vdash_{\bar{\nabla} \mathbf{L} \mathbf{P}^{d}} A^{*}
$$

Proof. Assume that $\Theta \nvdash_{\bar{\gamma} \mathbf{L} \mathbf{P}^{d}} A^{*}$, where $A^{*}$ is got from $A$ by inductively replacing every atomic subformula $C\left(y / f_{B}(\bar{t})\right)$ of $A$, where $C(y)$ and $\bar{t}$ do not contain any Skolem functions, with the formula $\exists y(B(\bar{t}, y) \wedge C(y))$. By the completeness theorem for $\bar{\nabla} \mathbf{L} \mathbf{P}^{d}$ (Fact 1) it follows that there is some model $\mathfrak{A}_{\mathfrak{s}}$ such that $\mathfrak{A}_{\mathfrak{s}} \vDash \Theta$ and $\mathfrak{A}_{\mathfrak{s}}\left(A^{*}\right)=\perp$. Extend $\mathfrak{A}_{\mathfrak{s}}$ to a model $\mathfrak{H}_{\mathfrak{s}}^{s f_{\omega}}$ of $\Theta^{s f_{\omega}^{\supset}}$, where $\Theta^{s f_{\omega}^{\supset}}$ is obtained from $\Theta$ by the obvious modification of the method in Thm. 9. By construction, $\mathfrak{A}_{\mathfrak{s}}^{s f_{\omega}}\left(A^{*}\right)=\perp$. From Cor. 26 it now follows that $\mathfrak{P}_{\mathfrak{s}}^{s f_{\omega}}\left(A^{*}\right) \leq \mathfrak{A}_{\mathfrak{s}}^{s f_{\omega}}(A)$, and therefore that $\mathfrak{H}_{\mathfrak{s}}^{s f_{\omega}}(A)=\perp$. From the soundness theorem for $\bar{\nabla} \mathbf{L} \mathbf{P}^{d}$ it follows that $\Theta^{s f_{\omega}^{\supset}} K_{\bar{\gamma} \mathbf{L} \mathbf{P}^{d}} A$. Since it is obvious that

$$
\Theta^{s f f_{\omega}^{\supset}} \vdash_{\bar{\gamma} \mathbf{L} \mathbf{P}^{d}} A \Longleftrightarrow \Theta \vdash_{\bar{\nabla} \mathbf{L} \mathbf{P}^{d}\left[\mathcal{S}^{\supset}\right]} A
$$

we can conclude that $\Theta \nvdash_{\overline{\bar{\gamma}_{\mathbf{L}}} \mathbf{P}^{d}\left[\mathcal{S}^{\supset}\right]} A$ which ends the proof.

Notice that it follows from Lem. 27 that $\Theta \vdash_{\bar{\gamma} \mathbf{L} \mathbf{P}^{d}} A^{*} \Rightarrow \Theta \vdash_{\bar{\gamma} \mathbf{L} \mathbf{P}^{d}\left[\mathcal{S}^{\supset}\right]} A$ fails for the translation used in Thm. 28. The other obvious translation would be to replace $B\left(f_{A}(t)\right)$ by $\forall y(A(t, y) \supset B(y))$. However, it is easy to see that the model in Lem. 27 also assigns $\mathbf{b}$ to $\forall y(A(x, y) \supset B(y))$ for any variable assignment function, and so this translation would not fare any better. This is not to say that there are no translations such that $\Theta \vdash_{\bar{\nabla}} \mathbf{K}_{3}^{d} A^{*} \Leftrightarrow \Theta \vdash_{\bar{\nabla}} \mathbf{K}_{3}^{d}\left[\mathcal{S}^{\supset}\right]$ $A$ does hold. I have, however, not been able to come up with any other viable alternatives and doubt that there is one.

I will now show that $\bar{\nabla} \mathbf{L} \mathbf{P}^{d}$ is unfit for Skolem functions relative to both definitions of $\exists$ !. Fig. 1 showed that $\exists !^{\supset}$ is the stronger definition of $\exists$ ! in the sense that it entails the other in $\bar{\nabla} \mathbf{L} \mathbf{P}^{d}$. Because of this, the Skolemizer and the deSkolemizer relativized to it are the weakest versions of these rules 
and so it will suffice to show that $\bar{\nabla} \mathbf{L} \mathbf{P}^{d}$ is unfit for Skolem functions relative to $\exists !^{\supset}$.

Theorem 29. Adding either of the two rules

$$
\begin{array}{ll}
\text { (deSkỏemizer) } & \forall \bar{x} \exists ! y A(\bar{x}, y), \psi_{s \doteq f_{A}(\bar{t})} \vdash \psi_{A(\bar{t}, s)} \\
\text { (Skỏlemizer) } & \forall \bar{x} \exists ! y A(\bar{x}, y), \psi_{A(\bar{t}, s)} \vdash \psi_{s=f_{A}(\bar{t})}
\end{array}
$$

to $\bar{\nabla} \mathbf{L} \mathbf{P}^{d}[\mathcal{S}]$ yields a non-conservative extension.

Proof. (deSkỏlemizer) Let $\Theta:=\{\forall x \forall y(y \not x x), \forall x \exists ! y A(x, y)\}$, and let $f_{A}(x)$ be the Skolem function for $\forall x \exists ! y A(x, y)$. From $\forall x \forall y(y \neq x), \bar{\nabla} \mathbf{L} \mathbf{P}^{d}[\mathcal{S}]$ yields $\forall x \forall y\left(y \neq f_{A}(x)\right)$, and so the deSkỏlemizer suffices for deriving $\forall x \forall y(\neg A(x, y))$.

The $\bar{\nabla} \mathbf{L} \mathbf{P}^{d}$-countermodel, $\mathfrak{A}_{\mathfrak{s}}$, to $\forall x \forall y(\neg A(x, y))$ is as follows: let $|\mathfrak{A}|$ be the set $\{a, b\}$, and let $\mathfrak{s}(x)=a$ for every variable $x$. $\doteq$ and the non-logical predicate $A(x, y)$ are interpreted according to the following two matrices:

$$
\begin{aligned}
& \begin{array}{c|ccc|cc}
\doteq & a & b
\end{array} \quad \begin{array}{llll}
A & a & b \\
\hline a & \mathbf{b} & \perp
\end{array} \quad \begin{array}{llll}
a & \top & \perp
\end{array} \\
& b|\perp \quad b \quad b| \perp \top
\end{aligned}
$$

It is easy to check that $\mathfrak{A}_{\mathfrak{s}}$ is in fact a model for $\Theta$. Expand $\mathfrak{A}_{\mathfrak{s}}$ into the model $\mathfrak{A}_{\mathfrak{s}}^{s f_{\omega}}$ of $\Theta^{s f_{\omega}}$ in accordance with Thm. 9. It is easy to see that $f_{A}^{2 \mathfrak{I}_{\mathfrak{s}}^{s f_{\omega}}}(a)=a$ and $f_{A}^{2 \mathfrak{U}_{\mathfrak{s}}^{s f_{\omega}}}(b)=b$, that $\mathfrak{U}_{\mathfrak{s}}^{s f_{\omega}} \vDash \forall x \forall y\left(y \neq f_{A}(x)\right)$, but that $\mathfrak{U}_{\mathfrak{s}}^{s f_{\omega}} \not$ $\forall x \forall y(\neg A(x, y))$. Thus $\mathfrak{A}_{\mathfrak{s}}^{s f_{\omega}}$ fails to validate deSkỏlemizer. $\forall x \forall y(\neg A(x, y))$ is a formula in the Skolem-function-free language, and so it follows by construction of $\mathfrak{A}_{\mathfrak{s}}^{s f_{\omega}}$ that $\mathfrak{A}_{\mathfrak{s}} \not \models \forall x \forall y(\neg A(x, y))$. By the soundness theorems for $\bar{\nabla} \mathbf{L P}^{d}$ it then follows that $\Theta \nvdash \forall x \forall y(\neg A(x, y))$ which shows that the addition of deSkỏlemizer yields a non-conservative extension.

(Skollemizer) The proof is similar to the previous one: let $\Theta$ be the set $\{\forall x \forall y A(x, y), \forall x \exists ! y A(x, y)\}$. The Skỏlemizer suffices for deriving $\forall x \forall y(x \doteq$ $y$ ) from $\Theta$. Modify the above model so that

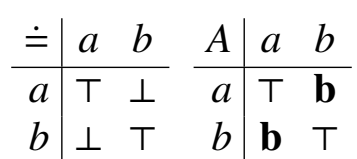

This model validates $\Theta$, but $\forall x \forall y(x \doteq y)$ fails in its Skolem extension. The same reasoning as above then shows that the addition of the Skolemizer yields a non-conservative extension in $\bar{\nabla} \mathbf{L} \mathbf{P}^{d}$.

Corollary 30. $\bar{\nabla} \mathbf{L} \mathbf{P}^{d}$ is unfit for Skolem functions relative to the two definitions of $\exists$ !.

I have in this section shown that there is a translation which allows one to eliminate definable Skolem functions while preserving derivability. It did 
not, however, preserve derivability when going from the original language to the extended language of definable Skolem functions. This is of course a drawback, but I think that one could in fact live well with it. The positive result for $\bar{\nabla} \mathbf{L} \mathbf{P}^{d}$ guarantees that any theorem derivable using the added bits of language, is actually a logical consequence of the axioms set forth. That some theorems are not translatable into the enriched language should not be of much concern; if it is, then one should rather introduce the function as a primitive function. I find it more awkward to give up the idea that one ought to be able to intersubstitute a Skolem equation $s \doteq f_{A}(\bar{t})$ and the formula $A(\bar{t}, s)$. The reason one can not do so in $\bar{\nabla} \mathbf{L} \mathbf{P}^{d}$ is basically because it has no conditional which obeys modus ponens; that $\supset$ does not obey modus ponens, makes it quite a strain to accept any of the two definitions of $\exists$ ! using it as credible.

Both $\bar{\nabla} \mathbf{K}_{3}^{d}$ and $\bar{\nabla} \mathbf{L P}^{d}$ do moderately well with regards to Skolem functions; it seems beyond a doubt that $\bar{\nabla} \mathbf{K}_{3}^{d}$ outperforms $\bar{\nabla} \mathbf{L} \mathbf{P}^{d}$, but one should note that $\forall \bar{x} \exists ! y A+\forall \bar{x} \forall y(\neg A \vee A)$. Thus $\bar{\nabla} \mathbf{K}_{3}^{d}$ can only express unique existence for classical formulas. It seems therefore attractive to investigate whether there are non-classical logics with conditionals which obeys both identity $(A \rightarrow A)$, modus ponens and have the expressive resources to define $\exists$ ! in a more credible way which does not preclude non-classicality from the outset. The next section looks at relevant logics with this in mind.

\section{Definable Skolem functions in Relevant logics}

This section introduces the relevant logics and shows that they too are unfit for Skolem functions relative to a variety of definitions of $\exists$ !. I also show that despite this, there is a translation which preserves derivability for some of these logics.

Australasian Journal of Logic (14:1) 2017, Article no. 7 
The relevant logic $\bar{\nabla} \mathbf{B B}^{d t o}$ has the following axioms and rules:

\begin{tabular}{|c|c|c|}
\hline BBAx1 & $A \rightarrow A$ & \\
\hline BBAx2 & $A \rightarrow A \vee B$ and $B \rightarrow A \vee B$ & \\
\hline BBAx3 & $A \wedge B \rightarrow A$ and $A \wedge B \rightarrow B$ & \\
\hline BBAx4 & $\neg \neg A \rightarrow A$ & \\
\hline BBAx 5 & $A \wedge(B \vee C) \rightarrow(A \wedge B) \vee(A \wedge C)$ & \\
\hline BBR1 & $A, B \vdash A \wedge B$ & \\
\hline BBR2 & $A, A \rightarrow B \vdash B$ & \\
\hline BBR3 & $A \rightarrow B \vdash(B \rightarrow C) \rightarrow(A \rightarrow C)$ & \\
\hline BBR4 & $A \rightarrow B \vdash(C \rightarrow A) \rightarrow(C \rightarrow B)$ & \\
\hline BBR5 & $A \rightarrow \neg B \vdash B \rightarrow \neg A$ & \\
\hline BBR6 & $A \rightarrow B, A \rightarrow C \vdash A \rightarrow B \wedge C$ & \\
\hline BBR7 & $A \rightarrow C, B \rightarrow C \vdash A \vee B \rightarrow C$ & \\
\hline BBR8 & $A \dashv \mathbf{t} \rightarrow A$ & \\
\hline BBR9 & $(A \circ B) \rightarrow C \Vdash t A \rightarrow(B \rightarrow C)$ & \\
\hline BBQ1 & $\forall x A \rightarrow A(x / t)$ & $t$ free for $x$ \\
\hline BBQ2 & $\forall x(A \vee B) \rightarrow(A \vee \forall x B)$ & $x \notin F V\{A\}$ \\
\hline BBQ3 & $\forall x(A \rightarrow B) \vdash A \rightarrow \forall x B$ & $x \notin F V\{A\}$ \\
\hline BBQ4 & $A(x / t) \rightarrow \exists x A$ & $t$ free for $x$ \\
\hline BBQ5 & $A \wedge \exists x B \rightarrow \exists x(A \wedge B)$ & $x \notin F V\{A\}$ \\
\hline BBQ6 & $\forall x(B \rightarrow A) \vdash \exists x B \rightarrow A$ & $x \notin F V\{A\}$ \\
\hline E1 & $\forall x(x \doteq x)$ & \\
\hline E2 & $t \doteq s, A(x / t) \vdash A(x / s)$ & $s \& t$ free for \\
\hline
\end{tabular}

The logics $\bar{\nabla} \mathbf{B} \mathbf{B}^{d \mathrm{t}}$ and $\bar{\nabla} \mathbf{B} \mathbf{B}^{d}$ are got from $\bar{\nabla} \mathbf{B} \mathbf{B}^{d t o}$ by deleting, respectively, BBR9 and BBR8 \& BBR9. Since I will use the same definition of what a proof is, we automatically get RQ, MR1 and MR2. Some defined connectives:

\section{Definition 22.}

$$
\begin{aligned}
& A \leftrightarrow B:=(A \rightarrow B) \wedge(B \rightarrow A) \\
& A \mapsto B:=(A \wedge \mathbf{t}) \rightarrow B
\end{aligned}
$$

The semantics of $\overline{\bar{\gamma}} \mathbf{B B}^{d t o}$ is more complicated to describe. It will, however, suffice to notice that one of the models for the propositional fragment of $\bar{\nabla} \mathbf{B B}^{d t o}$ is Belnap's model of relevance, $\mathfrak{B}$. The propositional logic $\mathbf{B} \mathbf{B}^{d t o}$ is a relevant logic and as such it has the property that if $A \rightarrow B$ is a logical theorem where $A$ and $B$ do not contain propositional constants, then $A$ and $B$ share a propositional variable. To show this Nuel D. Belnap introduced in [5] the 8 -valued model shown in Fig. 2 in which $+0,+1,+2$ and +3 are all designated, $\neg$ and $\rightarrow$ are interpreted according to the displayed matrices and conjunction and disjunction are interpreted as infimum and supremum over the displayed ordering (a boolean algebra). $\circ$ is an intensional conjunction, 
often called fusion. Belnap's model of relevance evaluates $A \circ B$ to the same value as $\neg(A \rightarrow \neg B)$. The Ackermann constant $\mathbf{t}$ is evaluated to the least designated element, which in $\mathfrak{B}$ is $+0 .{ }^{12}$

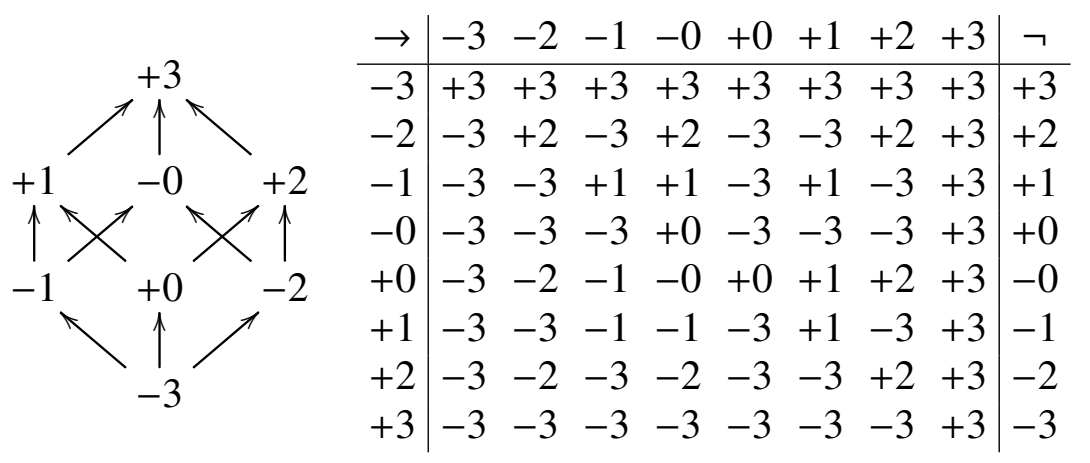

FIGURE 2. Belnap's model of relevance

Getting a model for first-order logic with identity from $\mathfrak{B}$ is quite standard: add a quantification domain $|\mathfrak{B}|$ and a variable assignment function $\mathfrak{s}$ and let $\mathfrak{B}_{\mathfrak{s}}$ interpret variables, names and function symbols in the same way a $\bar{\nabla} \mathbf{K}_{3}^{d}$-model does. The quantifiers are also in this case interpreted as infimum and supremum over the ordering of the valuespace. The only requirement upon the interpretation of the identity predicate is that for all $a, b \in|\mathfrak{B}|, \mathfrak{B}(\dot{\doteq})(a, b) \in\{+0,+1,+2,+3\} \Leftrightarrow a=b$.

The variable sharing property of relevant logics only applies to the propositional fragment and it is not evident how to extend it to deal with quantifiers and identity. It is, however, quite common to see relevantists deem formulas such as

$$
\text { (E5) } \forall x \forall y(x \doteq y \rightarrow(A \rightarrow A(x / y)))
$$

inappropriate since it entails $s \doteq t \rightarrow(A \rightarrow A)$ for any sentence $A$. The reason given is that there need be no relevant connection between $s \doteq t$ and $A \rightarrow A .^{13}$ The versions

$$
\text { (E6) } \forall x \forall y((x \doteq y \wedge A) \rightarrow A(x / y))
$$

\footnotetext{
${ }^{12}$ Belnap introduced this model as a model of the relevant logic $\mathbf{E}$ and remarked that it is also a model for Wilhelm Ackermann's logic $\Pi^{\prime}$ (first presented in [1]). $\Pi^{\prime}$ contained disjunctive syllogism (there called $\gamma$ ), that is $A, \neg A \vee B \vdash B$. Relevant logics are often assumed to be paraconsistent, that disjunctive syllogism fails in them; for instance, Stephen Read writes "[...] I claim that the rejection of DS $\vee$ [disjunctive syllogism], is central to the whole conception of relevant logic." ([18, p. 66]). Note, however, that $\gamma$, and even the stronger axiom $(A \wedge \neg A) \mapsto B$, holds true in Belnap's model of relevance.

${ }^{13}$ See for instance [17, p. 553].
}

Australasian Journal of Logic (14:1) 2017, Article no. 7 
is not subject to the same "irrelevant" counterexample as E5, and is sometimes taken to be relevantly permissible. Note that E6 can come out false in Belnap's model of relevance: let $|\mathfrak{B}|=\{a, b\}$ and let it interpret $A(x)$ and $\doteq$ according to the following matrices:

$$
\begin{aligned}
& \begin{array}{c|cc}
\doteq & a & b
\end{array} \quad \begin{array}{c|cc}
A & a & b \\
\hline a & +1 & -2
\end{array} \quad \begin{array}{ccc}
+0 & -3
\end{array} \\
& \text { b }-1+1
\end{aligned}
$$

It is then easy to calculate that $\mathfrak{B}_{5}\left(\forall x \forall y((x \doteq y \wedge A) \rightarrow A(x / y))=-3 \cdot{ }^{14}\right.$ I propose the following definition of a relevantly permissible version of Leibniz's law:

Definition 23. A version of Leibniz's law is RELEVANTLY PERMISSIBLE if every instance of it is evaluated to a designated value in any extension of Belnap's model of relevance into a model for quantified logic with the above restriction of the interpretation of $\doteq$.

The strongest axiomatic version of Leibniz's law which come out true on every way of extending $\mathfrak{B}$ into a model for first order logic with identity seems to be the following: ${ }^{15}$

$$
\text { (E7) } \forall x \forall y(A \rightarrow(x \doteq y \mapsto A(x / y))) \text {. }
$$

I will later show that there is a translation from the Skolem-extended language into the original one which preserves derivability in $\bar{\nabla} \mathbf{B B}^{d t}$ strengthened by E7 together with Ackermann's $\delta$ rule $-A \rightarrow(B \rightarrow C), B \vdash A \rightarrow C$.

The most obvious two ways of defining $\exists$ ! in relevant logics are as

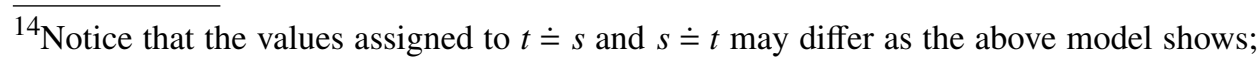
$\forall x \forall y(x \doteq y \mapsto y \doteq x)$ comes out true in every first-order model over Belnap's model of relevance, whereas $\forall x \forall y(x \doteq y \rightarrow y \doteq x)$ does not.

${ }^{15}$ See [14, Sec. 7] for a classification of versions of Leibniz's law in terms of strength and relevance. It is worth noting that $\mathrm{E} 4$ comes out as a relevantly permissible version of Leibniz's law on the above definition. Note also that

$$
\forall x_{1}, \ldots, x_{n} \forall y_{1}, \ldots, y_{n}\left(A\left(x_{1}, \ldots, x_{n}\right) \rightarrow\left(\bigwedge_{i=1}^{i=n} x_{i} \doteq y_{i} \mapsto A\left(y_{1}, \ldots, y_{n}\right)\right)\right)
$$

comes out true in Belnap's model of relevance, but that

$$
\forall x_{1}, \ldots, x_{n} \forall y_{1}, \ldots, y_{n}\left(A\left(x_{1}, \ldots, x_{n}\right) \rightarrow\left(\bigcirc_{i=1}^{i=n} x_{i} \doteq y_{i} \mapsto A\left(y_{1}, \ldots, y_{n}\right)\right)\right)
$$

does not, where $\bigcirc_{i=1}^{i=1} A_{i}:=A_{1}$, and $\bigcirc_{i=1}^{i=j+1} A_{i}:=\bigcirc_{i=1}^{i=j} A_{i} \circ A_{j+1}$ : simply consider the case when $n=2$ and give the following interpretation to $A$ and $\doteq$ :

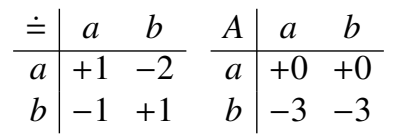

It is then easy to verify that $\forall x_{1} \forall x_{2} \forall y_{1} \forall y_{2}\left(A\left(x_{1}, x_{2}\right) \rightarrow\left(\left(x_{1} \doteq y_{1} \circ x_{2} \doteq y_{2}\right) \mapsto A\left(y_{1}, y_{2}\right)\right)\right)$ gets assigned the value -3 (instantiate $a$ for $x_{1}$ and $y_{2}$ and $b$ for $x_{2}$ and $y_{1}$ ).

Australasian Journal of Logic (14:1) 2017, Article no. 7 


\section{Definition 24.}

$$
\begin{aligned}
& \exists ! \vec{x} A:=\exists x(A \wedge \forall z(A(x / z) \rightarrow z \doteq x)) \\
& \exists ! \stackrel{x}{A} A:=\exists x \forall z(A(x / z) \leftrightarrow z \doteq x) .
\end{aligned}
$$

It is easy to show that $\exists ! \stackrel{x}{x} A+\exists ! \vec{x} A$ holds in $\bar{\nabla} \mathbf{B} B^{d}$ and that the converse is true if $\mathrm{E} 2$ is strengthened to $\mathrm{E} 8$ :

$$
\text { (E8) } A(x / t) \vdash \forall x(t \doteq x \rightarrow A) t \text { free for } x \text {. }
$$

I will first show that any relevant logic is fit for Skolem functions relative to the latter definition. However, I will argue that defining $\exists$ ! in this way makes it simply too hard to prove uniqueness claims. To remedy this one would have to add E8. This rule, however, entails E5 and is therefore not relevantly permissible. I then show that relevant logics are unfit for Skolem functions relative to the first definition and many variations thereof.

For logics such as $\bar{\nabla} \mathbf{B} B^{d}$ it is possible to prove that if $A$ and $B$ are alike with regards to free variables, then the rule $\forall \bar{x}(A \leftrightarrow B) \vdash \theta_{A} \leftrightarrow \theta_{B}$ is derivable, where $\theta_{B}$ is obtained from $\theta_{A}$ by replacing any number of $A$ 's with $B$ 's. Furthermore, since $A \leftrightarrow A$ holds in $\bar{\nabla} \mathbf{B B}^{d}$, it follows from both the Skolemizer and the deSkolemizer that $A(\bar{t}, s) \leftrightarrow s \doteq f_{A}(\bar{t})$. It follows that an extensions of $\bar{\nabla} \mathbf{B B}^{d}$ is, relative to a definition of $\exists$ !, fit for Skolem functions if and only it can be conservatively extended by the Skolem rule and the rule

$$
\text { (SkInt) } \forall \bar{x} \exists ! y A(\bar{x}, y) \vdash \forall \bar{x} \forall y\left(A(\bar{x}, y) \leftrightarrow y \doteq f_{A}(\bar{x})\right)
$$

and unfit otherwise. The focus will therefore be on this rule.

From the definition of $\exists ! \overleftrightarrow{x} A$ we straight away get that if $\Theta \vdash \forall \bar{x} \exists ! \overleftrightarrow{y} A$, then $\Theta^{s f} \vdash \forall \bar{x} \forall z\left(A(\bar{x}, z) \leftrightarrow z \doteq f_{A}(\bar{x})\right)$. We therefore have the following theorem: ${ }^{16}$

Theorem 31. $\bar{\nabla} \mathbf{B B}^{d}$ is fit for Skolem functions relative to $\exists !$.

Does this solve the problem? Whatever the answer it seems at least that it begets yet another problem: E8 is needed in order to derive $\forall \bar{x} \exists y \forall z(z \dot{\doteq} y \rightarrow$

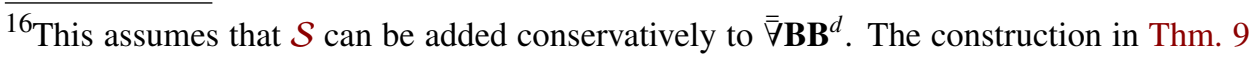
relied on the strong soundness and completeness theorems, and so it is worth noting that relevant logics can be generally be shown to be strongly sound and complete with regards to a certain algebraic semantics. For details, see [6], [7] and [19].
}

Australasian Journal of Logic (14:1) 2017, Article no. 7 
$A(\bar{x}, z))$ from $\forall \bar{x} \exists y A(\bar{x}, y)$ :

$\begin{array}{lll}\text { (1) } & \forall \bar{x} \exists y A(\bar{x}, y) & \text { assumption } \\ \text { (2) } & \exists y A(\bar{x}, y) & 1, \mathrm{Q} 1 \\ \text { (3) } & A(\bar{x}, v) & \text { assumption for MR2 } \\ \text { (4) } & v \doteq z \rightarrow A(\bar{x}, z) & 3, \mathrm{E} 8 \\ \text { (5) } & x / z \doteq z & \text { E1 } \\ \text { (6) } & z \doteq v \rightarrow x / v \doteq z & 5, \mathrm{E} 8 \\ \text { (7) } & z \doteq v \rightarrow A(\bar{x}, z) & 4,6, \text { transitivity of } \rightarrow \\ \text { (8) } & \forall z(z \doteq v \rightarrow A(\bar{x}, z)) & 7, \mathrm{RQ} \\ \text { (9) } & \exists y \forall z(z \doteq y \rightarrow A(\bar{x}, z)) & 7, \mathrm{BBQ} 4 \\ \text { (10) } \exists y \forall z(z \doteq y \rightarrow A(\bar{x}, z)) & 3-9, \mathrm{MR} 2 \\ \text { (11) } \forall \bar{x} \exists y \forall z(z \doteq y \rightarrow A(\bar{x}, z)) & 10, \mathrm{RQ}\end{array}$

E8 is a strong version of Leibniz's law. Since the problem of Skolem functions was raised by Weber in the context of naïve set theory, I should note that E8 is too strong in that setting; Andrew Bacon has recently shown that E8 turns naïve set theory trivial $([2, \S 2.2]) .{ }^{17}$ What is more dire for the relevantist, however, is that E8 makes the logic into an "irrelevant" one-it is easy to see that E8 entails the relevantly impermissible version of Leibniz's law E5:
(1) $A \rightarrow A(x / x)$
BBAx 1
(2) $\forall y(x \doteq y \rightarrow(A \rightarrow A(x / y))) \quad 1, \mathrm{E} 8$
(3) $\forall x \forall y(x \doteq y \rightarrow(A \rightarrow A(x / y))) \quad 2, \mathrm{RQ}$

Thus it seems contrary to the doctrine of relevant logics to demand that E8 should be a rule of logic. I therefore take it that $\exists ! !^{\oplus}$ is an inappropriate definition of $\exists$ ! for relevant logics as it makes it simply too hard to prove that $A(\bar{x}, y)$ is functional.

«Parenthetical remark. There is one possible argument for holding on to

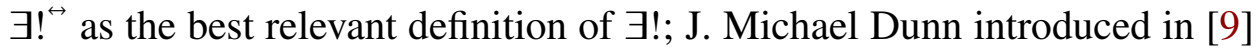
the notion of relevant predication where " $a$ relevantly has the property of being (an $x$ ) such that $A$ " is defined as

$$
(\varrho x A(x)) a:=\forall x(x \doteq a \rightarrow A(x)) .
$$

The relevantist could utilize this definition and define " $A(\bar{x}, y)$ is relevantly functional" to be

$$
\forall \bar{x} \exists y((\varrho z A(\bar{x}, z)) y \wedge \forall v(A(\bar{x}, v) \rightarrow v \doteq y)),
$$

which is easily seen to be nothing over and above $\forall \bar{x} \exists ! \overleftrightarrow{y} A(\bar{x}, y)$. Since other definitions of $\exists$ !, as we will see, do not secure that (SkInt) can be conservatively added, the relevantist could argue that $A(\bar{t}, s)$ and $s \doteq f_{A}(\bar{t})$ ought not

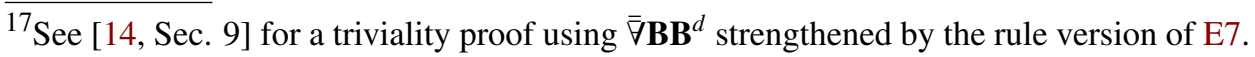
Australasian Journal of Logic (14:1) 2017, Article no. 7 
be intersubstitutable unless $A(\bar{x}, y)$ is relevantly functional. Providing such an argument is, however, a task better left to the relevantist. Let me note that it is somewhat surprising that Dunn, in explaining why "if anyone is Socrates then he is wise" is true while "if anyone is Reagan then Socrates is wise" is not, appeals E8 which he deems to be "presumptively at least, a relevantly valid argument" [9, p. 350]. Also, in iterating Dunn's example, Philip Kremer calls E8 "plausibly a relevant principle" in both [11, p. 350] and [12, p. 39]. ${ }^{18}$ Notice furthermore that Belnap's model of relevance can be used to show that Socrates can fail to be relevantly wise while at the same time validating "if anyone is Reagan then Socrates is wise": let the language consist of the names $s$ and $r$ together with the unary predicate Wise $(x)$. Let $|\mathfrak{B}|=\{$ Socrates, Reagan $\}$ and let $s^{\mathfrak{B}}=$ Socrates and $r^{\mathfrak{B}}=$ Reagan. Furthermore, let $W i s e(x)$ and $\doteq$ be interpreted according to the following matrices:

\begin{tabular}{|c|c|c|c|c|c|}
\hline$\doteq$ & Socrates & Reagan & Wise & Socrates & Reagan \\
\hline Socrates & +1 & -3 & & +2 & -2 \\
\hline Reagan & -3 & +2 & & & \\
\hline
\end{tabular}

It is then easy to calculate that $\mathfrak{B}_{\mathfrak{s}}(\forall x(x \doteq s \rightarrow$ Wise $(x)))=-3$ and $\mathfrak{B}_{\mathfrak{s}}(\forall x(x \doteq$ $r \rightarrow$ Wise $(s)))=+2$; that is "if anyone is Socrates then he is wise" comes out false, whereas both "Socrates is wise" and "if anyone is Reagan then Socrates is wise" comes out true. I take this to undermine the whole concept of relevant predication, but will not argue the issue further. End parenthetical.»

Since $\exists !^{\leftrightarrow}$ is an inappropriate definition of $\exists$ !, it seems worth while to

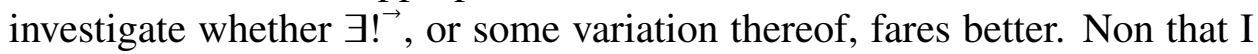
have been able to come up with do, as I will now show.

Theorem 32. If $\mathbf{L}$ is a first-order logic with identity extending $\bar{\nabla} \mathbf{B B}^{d}$ for which any way of extending Belnap's model of relevance into a model for first-order logic with identity is a model, then $\mathbf{L}$ is unfit for Skolem functions relative to $\exists !$.

Proof. Let $\Theta:=\{\forall x A(x, x), \forall x \exists ! \vec{y} A(x, y)\}$. If $\forall x \forall z\left(A(x, z) \leftrightarrow z \doteq f_{A}(x)\right)$ is added to $\Theta$, then $\forall x(A(x, x) \leftrightarrow x \doteq x)$ becomes derivable:

\footnotetext{
${ }^{18}$ Both Dunn and Kremer do make a note of the fact that
}

$$
\text { (E9) } \forall x \forall y(A \rightarrow(x \doteq y \rightarrow A(x / y)))
$$

has to fail in $\bar{\nabla} \mathbf{R}^{d}$. However, since $\bar{\nabla} \mathbf{R}^{d}$ validates the permutation rule $A \rightarrow(B \rightarrow C) \vdash$ $B \rightarrow(A \rightarrow C)$ it is easy to see that E9 is derivable from E8: as I showed above, E8 entails E5 from which the permutation rule yields E9.

Australasian Journal of Logic (14:1) 2017, Article no. 7 


$\begin{array}{ll}\text { (1) } \forall x \exists ! \vec{y} A(x, y) & \text { assumption } \\ \text { (2) } \forall x \forall z\left(A(x, z) \leftrightarrow z \doteq f_{A}(x)\right) & \text { 1, SkInt } \\ \text { (3) } A(x, x) \leftrightarrow x \doteq f_{A}(x) & \text { 2, Q1 } \\ \text { (4) } \forall x A(x, x) & \text { assumption } \\ \text { (5) } A(x, x) & \text { 4, Q1 } \\ \text { (6) } x \doteq f_{A}(x) & \text { 4, 5, BBR2 } \\ \text { (7) } A(x, x) \leftrightarrow x \doteq x & 3,6 \text {, E2 } \\ \text { (8) } \forall x(A(x, x) \leftrightarrow x \doteq x) & 1-7, \mathrm{RQ}\end{array}$

If $\mathbf{L}$ were fit for Skolem functions, this formula should be derivable without using SkInt, and should therefore, using the soundness theorem for $\mathbf{L}$ and the assumption of this theorem, be true in any way of extending $\mathfrak{B}$ into a $\Theta$-model. I will now show forth such a model in which it fails.

Let $\mathfrak{B}_{\mathfrak{s}}$ be Belnap's model of relevance with the quantification domain $\{a, b\}$, and let it interpret $A(x, y)$ and $\doteq$ according to the following tables:

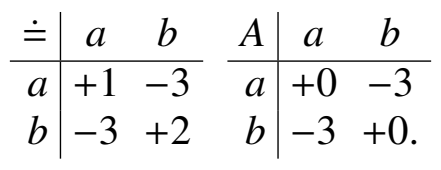

It is easy to verify that $\mathfrak{B}_{\mathfrak{s}}$ is in fact a model for $\Theta$, but that $\mathfrak{B}_{\mathfrak{s}}(\forall x(A(x, x) \leftrightarrow$ $x \doteq x))=-3$ which shows that the addition of $\forall x \forall z\left(A(x, z) \leftrightarrow z \doteq f_{A}(x)\right)$ makes for a non-conservative extension, and therefore that $\mathbf{L}$ is unfit for Skolem functions relative to $\exists ! \cdot$.

Thus using either of $\exists !^{\hookrightarrow}$ and $\exists ! \rightarrow$ has severe consequences: if $\exists ! !^{\rightarrow}$ is taken as the definition of $\exists$ !, then one can't intersubstitute $A(\bar{t}, s)$ and $s \doteq f_{A}(\bar{t})$, whereas taking $\exists$ ! $\mapsto$ as the definition of $\exists$ ! makes it exceedingly difficult to prove unique existence claims due to the absence of E8.

This is not to say that it is impossible to find a definition of $\exists$ ! such that the rule $\forall \bar{x} \exists ! y A(\bar{x}, y) \vdash \forall \bar{x} \forall y\left(A(\bar{x}, y) \leftrightarrow y \doteq f_{A}(\bar{x})\right)$ can be added conservatively. One possibility would be to use the defined connective $\mapsto$ instead of $\rightarrow$. $\mapsto$ is often utilized when defining restricted universal quantification for relevant logics so as to ensure that "every A is B" follows from "everything is B" ${ }^{19}$ It would therefore be natural to replace $\rightarrow$ with $\mapsto$ in $\exists !$, which would then be a natural translation of the English phrase "there exists one A which every A is identical to":

\section{Definition 25.}

$$
\exists ! \stackrel{\leftrightarrow}{x} A:=\exists x(A \wedge \forall z(A(x / z) \mapsto z \doteq x))
$$

\footnotetext{
${ }^{19}$ See [3] for a discussion of restricted quantification in relevant logics.
} 
However, since $A \rightarrow B \vdash A \mapsto B$ holds in Belnap's model of relevance, the non-conservativeness proof above would still work. This generalizes: if $\longmapsto$ is any conditional such that $A \rightarrow B \vdash A \longmapsto B$ holds in Belnap's model of relevance, then the above non-conservativeness proof also holds for $\exists !^{!} .^{20}$ Thus conditionals weaker than $\rightarrow$ will not do. ${ }^{21}$ One could consider stronger conditionals; for instance, the conditional $A \rightarrow B:=\top \rightarrow(A \rightarrow B)$, where $\mathrm{T}$ is the Church constant axiomatized by the axiom $A \rightarrow \mathrm{T}$, is strictly stronger than $\rightarrow ; A \rightarrow B \vdash A \rightarrow B$ fails in Belnap's model of relevance. Even so $\forall x \exists ! \vec{y} A(x, y)$ holds in the model, and so the non-conservativeness proof covers it too. Note also that replacing the extensional conjunction $\wedge$ with the intensional one $\circ$ would not help; the rule $A, B \vdash A \circ B$ is derivable using BBAx1 and BBR9, and so for any two operators $\nabla$ and $\partial$, if defining $\exists$ ! as $\exists x(\nabla(A) \wedge \partial(A(y), y \doteq x))$ does not work, then neither will $\exists x(\nabla(A) \circ \partial(A(y), y \doteq x))$. One could also try to replace $\leftrightarrow$ with some other biconditional in $\exists !^{\leftrightarrow}$. One such attempt would be $A \leadsto B:=A \leftrightarrow(B \wedge \mathbf{t})$. Note, however, that $\forall x \exists ! \stackrel{\leftrightarrow x}{y} A(x, y)$, unlike $\forall x \exists ! \stackrel{\leftrightarrow}{y} A(x, y)$, holds in the above model, and so the non-conservativeness proof covers it too.

There are undoubtedly many other ways to define $\exists$ ! and it might be that one of them is strict enough to ensure that (SkInt) can be added conservatively while at the same time not making it needlessly difficult to prove unique existence claims. I doubt that this is possible, but at this point I can do no better than to leave the finding of such a definition as a challenge for the relevantist.

Note that the rule version of E7-a relevantly permissible version of Leibniz's law—suffices for making $\forall \bar{x} \exists y A(\bar{x}, y) \vdash \forall \bar{x} \exists y \forall z(z \doteq y \mapsto A(\bar{x}, z))$ derivable. ${ }^{22}$ Thus if one defines $\exists$ ! as $\exists !^{m »}$, then any logic with the rule version of E7 will be able to prove uniqueness claims when appropriate, while at the same time having the following version of (SkInt) derivable:

$$
(\operatorname{SkInt} \leadsto) \forall \bar{x} \exists ! \stackrel{y}{y} A(\bar{x}, y) \vdash \forall \bar{x} \forall y\left(A(\bar{x}, y) \leftrightarrow y \doteq f_{A}(\bar{x})\right)
$$

This then shows that it is possible to at least intersubstitute $A(\bar{t}, s)$ and $s \doteq f_{A}(\bar{t}) \wedge \mathbf{t}$ when $A$ is $\leadsto$-functional. The question therefore is whether E2 can be strengthened to E7 without making the addition of the Skolem rule

\footnotetext{
${ }^{20}$ For instance, $A \rightarrow B \vdash A \supset B$ holds in the Belnap model of relevance-for all $i, j$ there is some $k$ such that $+i \rightarrow-j=-k$.

${ }^{21}$ One of the restrictions on restricted universal quantification set fourth by Beall et. al. in [3] is that $\forall x(A(x) \rightarrow B(x)) \vdash \Pi x(A(x), B(x))$ should hold where the binary quantifier $\Pi$ is the restricted universal quantifier. This then entails that relevant logics are unfit for Skolem functions relative to any way of defining the universal restricted quantifier provided $\exists ! x A$ is to be defined as $\exists x(A \wedge \Pi y(A(x / y), y \doteq x))$.

${ }^{22}$ That this is so is easily seen from the derivation above of $\forall \bar{x} \exists y \forall z(z \doteq y \rightarrow A(\bar{x}, z))$ from $\forall \bar{x} \exists y A(\bar{x}, y)$ using E8.
}

Australasian Journal of Logic (14:1) 2017, Article no. 7 
non-conservative. The following theorem shows that this is at least the case for $\mapsto$-definable Skolem functions:

Lemma 33. The rule

$$
\forall \bar{x} \exists ! \stackrel{y}{y} A(\bar{x}, y) \vdash \forall \bar{x}\left(B\left(f_{A}(\bar{x})\right) \leftrightarrow \forall y(A(\bar{x}, y) \mapsto B(y))\right),
$$

where $B(y)$ is any formula in which $f_{A}(\bar{x})$ is substitutable for $y$, is derivable in $\bar{\nabla} \mathbf{B B}^{d \mathbf{t}}\left[\mathcal{S}^{\mapsto}, \delta, E 7\right]-\bar{\nabla} \mathbf{B B}^{d \mathbf{t}}$ augmented with

$$
\begin{array}{cl}
\left(\mathcal{S}^{\mapsto}\right) & \forall \bar{x} \exists ! \stackrel{\leftrightarrow}{y} A(\bar{x}, y) \vdash \forall \bar{x}\left(A\left(\bar{x}, f_{A}(\bar{x})\right) \wedge \forall z\left(A(\bar{x}, z) \mapsto z \doteq f_{A}(\bar{x})\right)\right) \\
(\delta) & A \rightarrow(B \rightarrow C), B \vdash A \rightarrow C \\
(E 7) & \forall x \forall y(A \rightarrow(x \doteq y \mapsto A(x / y)))
\end{array}
$$

Proof. The following proof makes use of the derivable rules:

$$
\begin{aligned}
& \text { (I) } A \rightarrow(B \mapsto C), D \mapsto B \vdash A \rightarrow(D \mapsto C) \\
& \text { (II) } A \vdash A \wedge \mathbf{t} .
\end{aligned}
$$
(1) $\forall \bar{x} \exists y(A(\bar{x}, y) \wedge \forall z(A(\bar{x}, z) \mapsto z \doteq y))$
assumption
(2) $\forall \bar{x}\left(A\left(\bar{x}, f_{A}(\bar{x})\right) \wedge \forall z\left(A(\bar{x}, z) \mapsto z \doteq f_{A}(\bar{x})\right)\right)$
$1, \mathcal{S}^{\mapsto}$
(3) $\forall z(A(\bar{x}, z) \mapsto B(z)) \rightarrow\left(A\left(\bar{x}, f_{A}(\bar{x})\right) \mapsto B\left(f_{A}(\bar{x})\right)\right)$
(4) $A\left(\bar{x}, f_{A}(\bar{x})\right) \wedge \mathbf{t}$
(5) $\forall y(A(\bar{x}, y) \mapsto B(y)) \rightarrow B\left(f_{A}(\bar{x})\right)$
$\mathrm{BBQ} 1$
(6) $B\left(f_{A}(\bar{x})\right) \rightarrow\left(f_{A}(\bar{x}) \doteq y \mapsto B(y)\right)$
2, BBQ1 \& II
$3,4, \delta$
(7) $A(\bar{x}, y) \mapsto y \doteq f_{A}(\bar{x})$
E7 \& BBQ1
(8) $x / y \doteq y \rightarrow\left(y \doteq f_{A}(\bar{x}) \mapsto f_{A}(\bar{x}) \doteq y\right)$
2, BBQ1
(9) $\quad A(\bar{x}, y) \mapsto f_{A}(\bar{x}) \doteq y$
E7
(10) $B\left(f_{A}(\bar{x})\right) \rightarrow(A(\bar{x}, y) \mapsto B(y))$
7,8 , fiddling
(11) $B\left(f_{A}(\bar{x})\right) \rightarrow \forall y(A(\bar{x}, y) \mapsto B(y))$
$6,9, \mathrm{I}$
(12) $B\left(f_{A}(\bar{x})\right) \leftrightarrow \forall y(A(\bar{x}, y) \mapsto B(y))$
$10, \mathrm{RQ} \& \mathrm{BBQ} 3$
(13) $\forall \bar{x}\left(B\left(f_{A}(\bar{x})\right) \leftrightarrow \forall y(A(\bar{x}, y) \mapsto B(y))\right)$
$5,11, \mathrm{BBR} 1$
$12, \mathrm{RQ}$

The above lemma used $\exists !^{\mapsto}$ as the definition of $\exists$ !. However, since

$$
\begin{aligned}
& \exists ! \stackrel{\leftrightarrow}{y} A(y) \vdash \exists ! \vec{y} A(y) \\
& \exists ! \stackrel{\leftrightarrow}{y} A(y)+\exists ! \vec{y} A(y)
\end{aligned} \exists ! \vec{y} A(y) \vdash \exists ! \stackrel{\leftrightarrow}{y} A(y)
$$

the result also holds for either of the other three definitions of $\exists$ ! (and of course any definition $\exists !^{-\circ}$ of $\exists$ ! such that $\left.\exists ! y A(y)+\exists ! \dot{y} A(y)\right)$.

Theorem 34. $\bar{\nabla} \mathbf{B B}{ }^{d \mathbf{t}}\left[\mathcal{S}^{\mapsto}, \delta, E 7\right]$ conservatively extends $\bar{\nabla} \mathbf{B} \mathbf{B}^{d \mathbf{t}}[\delta, E 7]$.

Proof. Let $\mathcal{L}$ be the Skolem function free language, and $\mathcal{L}_{s_{\omega}}$ the Skolemenriched language. Furthermore, let $E 7 \uparrow_{\mathcal{L}}$ be $E 7$ restricted to $\mathcal{L}$. Assume that $\Theta{\left.\nvdash_{\bar{\nabla}} \mathbf{B B}^{d}[\delta, E\rceil_{\mathcal{L}}\right]} C$ for some formula $C \in \mathcal{L}$. The goal is to show that 
$\Theta \nvdash_{\bar{\nabla} \mathbf{B} \mathbf{B}^{d}\left[\mathcal{S}^{\mapsto, \delta, E 7]}\right.} C$. By the completeness theorem there is a model $\mathfrak{A}_{\mathfrak{s}}$ which validates $\Theta$, but not $C$. Extend $\mathfrak{H}_{\mathfrak{5}}$ to $\mathfrak{H}_{\mathfrak{5}}^{s_{f_{\omega}}}$ in line with Thm. 9 so as to validate the $\mapsto$-functional Skolem rule. $\quad \mathfrak{H}_{s}^{s f_{\omega}}$ obviously validates $E 7 \uparrow_{\mathcal{L}}$. Now for every instance $\psi:=\forall x \forall y(C \rightarrow(x \doteq y \mapsto C(x / y)))$ of E7 over $\mathcal{L}_{S_{\omega}}$, inductively replace every atomic subformula $B\left(f_{A}(\bar{t})\right)$ by the formula $\forall z(A(\bar{t}, z) \mapsto B(z))$, where both $\bar{t} \in \mathcal{L}$ and $B(z) \in \mathcal{L}$ and $z$ is a variable which does not occur in $\psi$. Note that $B(z)$ is a $\mathcal{L}$-formula. Let the resulting formula be $\psi^{*}$. $\psi^{*}$ is obviously an instance of $E 7 \uparrow_{\mathcal{L}}$, and so holds in $\mathfrak{H}_{s}^{s f_{\omega}}$. Notice furthermore that one only needs $E 7 \uparrow_{\mathcal{L}}$ in Lem. 33 in order to derive $\forall \bar{x}\left(D\left(f_{E}(\bar{x})\right) \leftrightarrow \forall y(E(\bar{x}, y) \mapsto D(y))\right)$ so long as $D$ is in $\mathcal{L}$. Thus $E 7 \uparrow_{\mathcal{L}}$ suffices for deriving the sentence $\forall \bar{x}\left(B\left(f_{A}(\bar{x})\right) \leftrightarrow \forall z(A(\bar{x}, z) \mapsto B(z))\right)$. Since the intersubstitutivity rule $\forall \bar{x}(D \leftrightarrow E) \vdash \theta_{D} \leftrightarrow \theta_{E}$ holds, it follows that $\Theta \vdash_{\overline{7} \mathbf{B B}} \mathbf{B}_{\left.[\mathcal{S} \leftrightarrow, \delta, E\rceil_{\mathcal{L}}\right]} \psi \leftrightarrow \psi^{*}$, and since $\psi^{*}$ is an $E 7 \Gamma_{\mathcal{L}^{-i n s t a n c e, ~ i t ~ f o l-~}}$ lows by modus ponens that $\Theta \vdash_{\bar{\gamma}} \mathbf{B B}^{d}\left[\mathcal{S}^{\left.\mapsto, \delta, E 7 \Gamma_{\mathcal{L}}\right]} \psi\right.$. Thus $\mathfrak{M}_{\mathfrak{s}}^{s f_{\omega}}$ also validates E7 over the full language $\mathcal{L}_{s_{\omega}}$. By the soundness theorem it now follows that $\Theta \nvdash_{\left.\bar{\nabla} \mathbf{B B} \mathbf{B}^{d}\left[\mathcal{S}^{\natural}, \delta, E\right\urcorner\right]} C$.

I have shown in this section that if one is willing to add E7, a rather strong, but relevant, version of Leibniz's law, then one may translate back and forth between the definable Skolem function extended language and the original language while preserving derivability. However, I think that the best result with regards to intersubstituting $s \doteq f_{A}(\bar{t})$ and $A(\bar{t}, s)$ one may hope for when applying a relevant logic is that $s \doteq f_{A}(\bar{t}) \wedge \mathbf{t}$ and $A(\bar{t}, s)$ may be instersubstituted.

\section{A BRIEF GLANCE AT OTHER LOGICS}

What of non-classical logics other than relevant logics? $\bar{\nabla} \mathbf{L} \mathbf{P}^{d}$ and $\bar{\nabla} \mathbf{K}_{3}^{d}$ have two natural extensions, namely the three-valued logics $\bar{\nabla} \mathbf{R} \mathbf{M}_{3}^{d}$ and $\bar{\nabla} \mathbf{L}_{3}^{d}$. A model for $\bar{\nabla} \mathbf{R} \mathbf{M}_{3}^{d} / \overline{\bar{\gamma}} \mathbf{L}_{3}^{d}$ is got from a $\bar{\nabla} \mathbf{L} \mathbf{P}^{d} / \bar{\nabla} \mathbf{K}_{3}^{d}$-model by interpreting the conditional $\rightarrow$ according to the following two matrices:

\begin{tabular}{|c|c|c|c|c|c|c|}
\hline$\stackrel{\mathrm{RM}_{3}}{\rightarrow}$ & $\mathrm{T}$ & b $\perp$ & $\stackrel{Ł_{3}}{\rightarrow}$ & T & n & $\perp$ \\
\hline$\top$ & $T$ & $\perp \perp$ & $T$ & $T$ & $\mathbf{n}$ & $\perp$ \\
\hline b & T & b $\perp$ & $\mathbf{n}$ & T & $\top$ & n \\
\hline$\perp$ & $T$ & $\begin{array}{cc}\top & \top\end{array}$ & $\perp$ & $\top$ & $\top$ & $T$ \\
\hline
\end{tabular}

$\bar{\nabla} \mathbf{R} \mathbf{M}_{3}^{d}$ is an extension of $\bar{\nabla} \mathbf{L} \mathbf{P}^{d}$, whereas $\bar{\nabla} \mathbf{L}_{3}^{d}$ is an extension of $\bar{\nabla} \mathbf{K}_{3}^{d}$. In both cases it can be shown that E8 fails. However, in both logics it is natural to define restricted universal quantification using a different conditionalin $\bar{\nabla} \mathbf{R} \mathbf{M}_{3}^{d}$ since $\rightarrow$ does not satisfy weakening; $A \nvdash B \rightarrow A$, and so $\forall x A \vdash$ $\forall x(B \rightarrow A)$ fails, in $\bar{\nabla} \mathbf{L}_{3}^{d}$ because it does not satisfy restricted modus ponens$\forall x(A \rightarrow(B \rightarrow C)), \forall x(A \rightarrow B)$ does not suffice for deriving $\forall x(A \rightarrow C)$ 
since contraction, the rule $A \rightarrow(A \rightarrow B) \vdash A \rightarrow B$, does not hold for $\rightarrow$ in $\bar{\nabla} \mathbf{L}_{3}^{d}$. There are two good candidates to overcome these difficulties, namely $A \rightarrow(A \rightarrow B)$ in the case of $\bar{\nabla} \mathbf{L}_{3}^{d}$ and $(A \rightarrow B) \vee B$, or equivalently $A \mapsto B$, in the case of $\bar{\nabla} \mathbf{R} \mathbf{M}_{3}^{d}$. Note also that one natural way to formulate Leibniz's law vernacularly is if $b$ is A, then everything identical to it is also $A$, which is most naturally formalized using restricted universal quantification: $\forall x(A \rightarrow \Pi y(x \doteq y, A(x / y)))$, where $\Pi$ is the, defined or not, restricted universal quantifier. It is also natural to use the settled upon definition of the restricted universal quantifier in order to define unique existential quantification as $\exists x(A \wedge \Pi y(A(x / y), y \doteq x))$. By defining $\Pi x(A, B)$ as $\forall x((A \rightarrow B) \vee B)$ in the case of $\bar{\nabla} \mathbf{R} \mathbf{M}_{3}^{d}$ and as $\forall x(A \rightarrow(A \rightarrow B))$ in the case of $\bar{\nabla} \mathbf{E}_{3}^{d}$, it is easy to see that $\forall x(A \rightarrow \Pi y(x \doteq y, A(x / y)))$ holds in any model. By modifying the proof in Lem. 33 ever so slightly it is then possible to prove that

$$
\forall \bar{x} \exists ! y A(\bar{x}, y) \vdash \forall \bar{x}\left(B\left(f_{A}(\bar{x})\right) \leftrightarrow \Pi y(A(\bar{x}, y), B(y))\right)
$$

is derivable in both logics. Thus both logics suffice for the existence of a translations which preserves derivability back and forth between the definable Skolem function extended language and the original one. However, neither of the logics can be conservatively extended by the rule

$$
\forall \bar{x} \exists ! y A(\bar{x}, y) \vdash \forall \bar{x} \forall y\left(A(\bar{x}, y) \leftrightarrow y \doteq f_{A}(\bar{x})\right)
$$

The proof of $\forall x(A(x, x) \leftrightarrow x \doteq x)$ in Thm. 32 also works in the case of $\bar{\nabla} \mathbf{R} \mathbf{M}_{3}^{d}$ and it is evident that there are countermodels to this formula also in $\bar{\nabla} \mathbf{R} \mathbf{M}_{3}^{d}$. We therefore have the following corollary:

Corollary 35. $\bar{\nabla} \mathbf{R} \mathbf{M}_{3}^{d}$ is unfit for Skolem functions relative to both the definitions $\exists x(A \wedge \forall y(A(x / y) \rightarrow y \doteq x))$ and $\exists x(A \wedge \forall y((A(x / y) \rightarrow y \doteq x)) \vee y \doteq x)$ of $\exists$ !.

There is a similar argument showing that $\bar{\nabla} \mathbf{L}_{3}^{d}$ is unfit for Skolem functions relative to both $\exists x(A \wedge \forall y(A(x / y) \rightarrow y \doteq x))$ and $\exists x(A \wedge \forall y(A(x / y) \rightarrow$ $(A(x / y) \rightarrow y \doteq x)))$ : first derive $\forall x \forall y(A(x, y) \vee(A(x, y) \leftrightarrow \neg A(x, y)))$ from the set $\Theta:=\{\forall x \exists ! y A(x, y), \forall x \forall y(x \doteq y \vee(x \doteq y \leftrightarrow x \neq y))\}$, where $\Pi x(A, B):=$ $\forall x(A \rightarrow(A \rightarrow B))$, using the rule

$$
\forall \bar{x} \exists ! y A(\bar{x}, y) \vdash \forall \bar{x} \forall y\left(A(\bar{x}, y) \leftrightarrow y \doteq f_{A}(\bar{x})\right) .
$$

The following model validates $\Theta$ and $\forall x \exists ! \vec{y} A(x, y)$, but fails to validate $\forall x \forall y(A(x, y) \vee(A(x, y) \leftrightarrow \neg A(x, y)))$ :

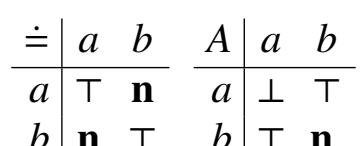

Australasian Journal of Logic (14:1) 2017, Article no. 7 
Corollary 36. $\bar{\forall} \mathbf{E}_{3}^{d}$ is unfit for Skolem functions relative to both the definitions $\exists x(A \wedge \forall y(A(x / y) \rightarrow y \doteq x))$ and $\exists x(A \wedge \forall y(A(x / y) \rightarrow(A(x / y) \rightarrow y \doteq x)))$ of $\exists$ !.

The moral seems to be this: if one wants to add definable Skolem functions as a mere façon de parler, that is as bits of language which one can translate into and away from while retaining any theorem, then one needs to find a way to express restricted universal quantification and use it to express unique existential quantification and Leibniz's law. However, if one demands that $\forall \bar{x} \exists ! y A(\bar{x}, y) \vdash \forall \bar{x} \forall y\left(A(\bar{x}, y) \leftrightarrow y \doteq f_{A}(\bar{x})\right)$ should be derivable or at least conservatively addable, then the safest bet would be to make sure that the logic validates E8.

\section{Summary}

This paper has shown how to conservatively extend a theory formulated in non-classical logics by a rule governing Skolem functions (Cor. 12). It was shown that this is possible in quite weak logics. I then showed that there is a translation for definable Skolem functions which preserves derivability in $\bar{\nabla} \mathbf{K}_{3}^{d}$ (Thm. 24), and to a lesser extent also in $\bar{\nabla} \mathbf{L} \mathbf{P}^{d}$ (Thm. 28). I showed that it matters greatly how one defines the unique existential quantifier in $\bar{\nabla} \mathbf{K}_{3}^{d}$ when it comes to what kind of reasoning one validly can do when confined to conservatively introduced Skolem functions. It was shown that $\bar{\nabla} \mathbf{L} \mathbf{P}^{d}$, even though the Skolem rule can be added conservatively to it, cannot validate the intersubstitutability of the equation $s \doteq f_{A}(\bar{t})$ and $A(\bar{t}, s)$ even though the latter formula is functional (Thm. 29). $\bar{\nabla} \mathbf{L} \mathbf{P}^{d}$ was for this reason deemed unfit for Skolem functions. $\bar{\nabla} \mathbf{K}_{3}^{d}$ was shown to fare better- $s \doteq$ $f_{A}(\bar{t})$ and $A(\bar{t}, s)$ can be intersubstituted conservatively if $\exists$ ! is defined as $\exists x \forall z(A(x / z) \equiv z \doteq x)$ (Thm. 17), and $A(\bar{t}, s)$ can be conservatively substituted for $s \doteq f_{A}(\bar{t})$ if $\exists$ ! is defined as $\exists x(A \wedge \forall z(A(x / z) \supset z \dot{\doteq} x))$ (Thm. 20). However, for this definition of $\exists$ ! it was shown that substituting $s \doteq f_{A}(\bar{t})$ for $A(\bar{t}, s)$ can result in non-conservativeness (Thm. 22).

I also showed that $\bar{\nabla} \mathbf{B B}^{d}$ is fit for Skolem functions provided that $\exists$ ! is defined as $\exists x \forall z(A(x / z) \leftrightarrow z \doteq x)$ ) (Thm. 31). However, defining $\exists$ ! this way makes it needlessly hard to prove unique existence claims due to the absence of the relevantly impermissible rule $A(x / t)+\forall x(t \doteq x \rightarrow A)$, E8. I furthermore showed that relevant logics are unfit for Skolem functions relative to the definition $\exists x(A(x) \wedge \forall y(A(y) \rightarrow y \doteq x)$ ) of $\exists$ ! (Thm. 32). A solution to the intersubstitutability problem in terms of relevant predication was suggested for the relevantist, although this theory was criticized for assuming E8. A translation which preserves derivability was also shown forth for certain relevant logics (Thm. 34). These two results was then shown to hold also for the three-valued logics $\bar{\nabla} \mathbf{R} \mathbf{M}_{3}^{d}$ and $\bar{\nabla} \mathbf{L}_{3}^{d}$ (Cor. 35 \& Cor. 36).

Australasian Journal of Logic (14:1) 2017, Article no. 7 


\section{REFERENCES}

[1] Wilhelm Ackermann. Begründung einer strengen Implikation. Journal of Symbolic Logic, 21:113-128, 1956. doi: 10.2307/2268750.

[2] Andrew Bacon. Paradoxes of logical equivalence and identity. Topoi, 34(1):89-98, 2015. doi: 10.1007/s11245-013-9193-8.

[3] Jc Beall, Ross T. Brady, A. P. Hazen, Graham Priest, and Greg Restall. Relevant restricted quantification. Journal of Philosophical Logic, 35(6):587-598, 2006. doi: 10.1007/s10992-005-9008-5.

[4] Jc Beall, Thomas Forster, and Jeremy Seligman. A note on freedom from detachment in the logic of paradox. Notre Dame Journal of Formal Logic, 54(1):15-20, 2013. doi: 10.1215/00294527-1731353.

[5] Nuel D. Belnap. Entailment and relevance. Journal of Symbolic Logic, 25(2):144146, 1960. doi: 10.2307/2964210.

[6] Ross T. Brady. A content semantics for quantified relevant logics I. Studia Logica, 47(2):111-127, 1988. doi: 10.1007/BF00370286.

[7] Ross T. Brady. A content semantics for quantified relevant logics II. Studia Logica, 48(2):243-257, 1989. doi: 10.1007/BF02770515.

[8] Petr Cintula and Petr Hájek. Triangular norm based predicate fuzzy logics. Fuzzy Sets and Systems, 161:311-346, 2010.

[9] J. Michael Dunn. Relevant predication 1: The formal theory. Journal of Philosophical Logic, 16(4):347-381, 1987. doi: 10.1007/BF00431183.

[10] Leon Henkin. The Completeness of the First-Order Functional Calculus. The Journal of Symbolic Logic, 14(3):159-166, 1949.

[11] Philip Kremer. Relevant predication: Grammatical characterisations. Journal of Philosophical Logic, 18(4):349-382, 1989. doi: 10.1007/BF00262941.

[12] Philip Kremer. Dunn's relevant predication, real properties and identity. Erkenntnis, 47(1):37-65, 1997. doi: 10.1023/A:1005306200547.

[13] Grigori Mints. Skolem's method of elimination of positive quantifiers in sequential calculi. Dokl. Akad. Nauk SSR, 169:861-864, 1966.

[14] Tore Fjetland Øgaard. Paths to triviality. Journal of Philosophical Logic, 45(3):237276, 2016. doi: 10.1007/s10992-015-9374-6.

[15] Terence Parsons. Indeterminate Identity: Metaphysics and Semantics. Oxford University Press, Oxford, 2000. doi: 10.1093/acprof:oso/9780198250449.001.0001.

[16] Graham Priest. The logic of paradox. Journal of Philosophical Logic, 8(1):219-241, 1979. doi: 10.1007/BF00258428.

[17] Graham Priest. An Introduction to Non-Classical Logic. From If to Is. Cambridge University Press, 2nd edition, 2008.

[18] Stephen Read. What is wrong with disjunctive syllogism? Analysis, 41(2):66-70, 1981. doi: $10.2307 / 3327233$.

[19] Greg Restall. On Logics Without Contraction. PhD thesis, The University of Queensland, 1994. Available at http://consequently.org/papers/onlogics.pdf.

[20] Joseph R. Shoenfield. Mathematical Logic. Addison-Wesley, 1967.

[21] Thoralf Skolem. Logico-combinatorial investigations in the satisfiability or provability of mathematical propositions: a simplified proof of L. Löwenheim and generalizations of the theorem. In Jean von Heijenoort, editor, From Frege to Gödel, A Source Book in Mathematical Logic, 1879-1931, pages 252-263. Harvard University Press, 1967.

Australasian Journal of Logic (14:1) 2017, Article no. 7 
[22] Craig Smoryński. The axiomatisation problem for fragments. Annals of Mathematical Logic, 14:193-221, 1978. doi: 10.1016/0003-4843(78)90016-5.

[23] Dirk van Dalen. Logic and Structure. Springer-Verlag, 4th edition, 2004.

[24] Zach Weber. Transfinite cardinals in paraconsistent set theory. The Review of Symbolic Logic, 5(2):269-293, 2012. doi: 10.1017/S1755020312000019.

\section{Appendix - Henkin axioms and IndePendence of Premise}

In many presentations of Henkin's completeness theorem one adds socalled Henkin axioms instead of Henkin witnesses; instead of expanding $\Theta$ to $\Theta^{h c}$ one rather adds every formula on the form $\exists x A(x) \rightarrow A\left(c_{A}\right)$. In classical logic this comes to the same thing. This is easily seen by noting that for every formula $\exists x A(x)$ and every model $\mathfrak{A}_{s}$, (a) if $\mathfrak{A}_{s} \vDash \exists x A(x)$, then $\mathfrak{A}_{s} \vDash A\left(c_{A}\right) \Rightarrow \mathfrak{A}_{s} \vDash \exists x A(x) \rightarrow A\left(c_{A}\right)$, and (b) if $\mathfrak{A}_{s} \not \vDash \exists x A(x)$, then $\mathfrak{A}_{s} \vDash \neg \exists x A(x)$, and so trivially $\mathfrak{A}_{s} \vDash \exists x A(x) \rightarrow A\left(c_{A}\right)$. However, (a) fails to be true for logics which do not validate the weakening rule $A \vdash B \rightarrow A$ such as relevant logics. (b) generally fails in logics with models where both $A$ and $\neg A$ can be assigned to a non-designated truth-value such as is the case with $\bar{\nabla} \mathbf{K}_{3}^{d}, \bar{\nabla} \mathbf{B B}{ }^{d \mathbf{t} \circ}$, intuitionistic logic and the fuzzy logic IMTL. ${ }^{23}$ The case with $\bar{\nabla} \mathbf{L} \mathbf{P}^{d}$ is somewhat special; it can be shown that one can add Henkin and Skolem axioms conservatively, but since modus ponens does not hold for its conditional, the interesting property is in this case that the rules can be added conservatively.

It is natural to think that the adding of Henkin/Skolem axioms and the Henkin/Skolem rule come apart because of a lack of a deduction theorem on the form $\Gamma, A \vdash B \Leftrightarrow \Gamma \vdash A \rightarrow B$. Such a deduction theorem fails in relevant logics, $\bar{\nabla} \mathbf{L} \mathbf{P}^{d}$ and $\bar{\nabla} \mathbf{K}_{3}^{d}$. Note, however, that it holds in intuitionistic logic, yet intuitionistic logic can only be conservatively extended by the Henkin/Skolem rules and not by their axiomatic counterparts.

The proof given in this essay that one may conservatively add the Henkin and Skolem rules conservatively applies to a range of logics for which the same is not the case with Henkin and Skolem axioms. For some of these logics, however, there is a simple property which suffices for making such an axiomatic extension conservative. I will now show that if we assume that the logic in question is sound and complete with regards witnessed models and validates some rather innocuous rules, then one may conservatively extend $\Theta$ by Henkin axioms if and only if $\Theta$ is logically closed under the strong linearity rule called Independence of Premise:

$$
\text { (IoP) } A \rightarrow \exists x B \vdash \exists x(A \rightarrow B) \quad x \notin F V(A),
$$

where $\Theta$ is logically closed under the rule just if $\Theta \vdash_{\mathbf{L}} A \rightarrow \exists x B$ entails that $\Theta \vdash_{\mathbf{L}} \exists x(A \rightarrow B)$, where $x$ is not a free variable of $A$.

\footnotetext{
${ }^{23}$ For a presentation of the latter, see [8].
}

Australasian Journal of Logic (14:1) 2017, Article no. 7 
Besides being a derivable rule in classical logic, IoP is also derivable in Łukasiewicz infinite-valued logic and in Gödel-Dummett logic. It is not, however, derivable in intuitionistic logic, relevant logics, nor in the fuzzy logic IMTL.

\section{Definition 26.}

$$
\left.\Theta_{a}:=\left\{\exists x B(x) \rightarrow B\left(x / c_{B}\right) \mid B \in \mathcal{L}\right\}\right)
$$

$\Theta^{\text {hae }}:=\Theta_{a} \cup \Theta$ is called the HeNKIN AXIOM EXTENSION of $\Theta$ and $\exists x B(x) \rightarrow$ $B\left(x / c_{B}\right)$ is called $a$ HENKIN AXIOM.

Theorem 37. If $\Theta$ is formulated in a logic which is sound and complete with regards to witnessed models and validates

$$
\begin{array}{lll}
(Q 3) & A(x / t) \vdash \exists x A & \text { t free for } x \\
\text { (BBAxl) } & A \rightarrow A & \\
\text { (transitivity) } & A \rightarrow B, B \rightarrow C \vdash A \rightarrow C, &
\end{array}
$$

then $\Theta^{\text {hae }}$ is a conservative extension of $\Theta$ if and only if $\Theta$ is logically closed under IoP.

Proof. $[\Longrightarrow]$ Assume that $\Theta$ can be conservatively extended by Henkin axioms and that $\Theta \vdash A \rightarrow \exists x B(x)$ where $x \notin F V(A)$.

$$
\begin{array}{ll}
\text { (1) } A \rightarrow \exists x B(x) & \text { assumption } \\
\text { (2) } \exists x B(x) \rightarrow B\left(c_{B}\right) & \text { Henkin axiom } \\
\text { (3) } A \rightarrow B\left(c_{B}\right) & 1,2 \text {, transitivity } \\
\text { (4) } \exists x(A \rightarrow B(x)) & 3, \mathrm{Q} 3
\end{array}
$$

Since $\Theta$ is extended conservatively and $\exists x(A \rightarrow B(x))$ is a formula in the language of $\Theta$, it follows that $\Theta$ is logically closed under IoP.

$[\Longleftarrow]$ Assume that that $\Theta$ is logically closed under IoP and that $\Theta \nvdash A$ for some $A$ in the language of $\Theta$. The goal is to show that $\Theta^{\text {hac }} \nvdash A$. The completeness theorem entails that there is a model $\mathfrak{A}_{\mathfrak{s}}$ such that $\mathfrak{A}_{\mathfrak{s}} \vDash \Theta$ and $\mathfrak{A}_{\mathfrak{s}} \not \models A$. By assumption of BBAx1 and IoP we have that for every formula $B, \mathfrak{A}_{\mathfrak{s}} \vDash \exists x(\exists x B(x) \rightarrow B(x))$. Since $\mathfrak{A}_{\mathfrak{s}}$ is witnessed it follows that for some $b \in|\mathfrak{A}|, \mathfrak{A}_{\mathfrak{s}(x / b)} \vDash \exists x B(x) \rightarrow B(x)$. So for every formula $B$ we have that the set $W^{B}:=\left\{b \in|\mathfrak{A}| \mid \mathfrak{A}_{\mathfrak{s}(x / b)} \vDash \exists x B(x) \rightarrow B(x)\right\} \not \emptyset$. By the axioms of choice there is a function which picks one element from each set $W^{B}$. Let this choice function determine the denotation of the Henkin constants $c_{B}$ and let $\mathfrak{A}_{s}^{\text {hae }}$ be got from $\mathfrak{A}_{\mathfrak{s}}$ by adding this interpretation. $\mathfrak{A}_{s}^{\text {hae }}$ is obviously a model for $\Theta^{\text {hae }}$. The value that $\mathfrak{U}_{s}^{\text {hae }}$ assigns to $A$ depends only on the objects assigned to the terms of $A$ and the values assigned to the atomic subformulas of $A$. Since $c_{B}$ does not occur in $A$ it follows that $\mathfrak{A}_{s}^{\text {hae }}$ has to assign $A$ the same value as $\mathfrak{A}_{\mathfrak{s}}$ and so $\mathfrak{A}_{s}^{h a e} \not k A$. It follows now from the soundness theorem that $\Theta^{\text {hae }} \nvdash A$. 
University of Oslo, Department of Linguistics and Scandinavian Studies

E-mail address: toreog@mail.uio.no

Australasian Journal of Logic (14:1) 2017, Article no. 7 